\title{
LES LAMELLIBRANCHES
}

recueillis dans les courses du "Willem Barents," durant les mois de Mai à Septembre 1880 et 1881

par le

Dr. J. T玨 OATIID

Professeur à l'Ecole moyenne supérieure d'Arnhem. 


\section{1e Partie. Systématique.}

Fam. Anomiidae.

\section{Anomia ephippium, L. (forma typica).}

L'exemplaire de la plus grande taille avait une longueur de $=10$ millim. $(\mathrm{L}=10 \mathrm{millim}$. $)$ et une hauteur de 8 millim. $\left.(\mathrm{A}=8 \text { millim. })^{\mathfrak{1}}\right)$.

Bibliographie. Lrnn. Syst. Nat. page 1150.

Fonbes and Hancock. Brit. Moll. II. pag. 325. Pl. LV. fig. 2, 3, 5, 7 et Pl. T, fig. 2.

Jefrereys. Brit. Conch. II. pag. 30 et V. Pl. XX, fig. 1, 1a et $1 \mathrm{~b}$.

A. Govld. Report on the Invertebrata of Massachusets, second Ed. pag. 204.

G. O. Sars. Moll. reg. Arct. Norvegia. pag. 14.

Synonymie. O streum parvum, List.

Dragages. Station IX. 1881.

Deux exemplaires ont été trouvés sur Waldheimia et huit sur Pecten Islandicus. MÜLLER.

Distribution géographique. La mer glaciale et les côtes de la Scandinavie, la mer du Nord et la mer Baltique, les côtes de la France et des îles Britanniques, la Mediterranée, les côtes de Madère, les côtes septentrionales, orientales et occidentales de l'Amérique.

Fam. Pectinidae.

2. Pecten groenlandicus, SowŁrby.

$$
\mathrm{L}=22, \mathrm{~A}=21, \mathrm{La}=4 .
$$

Bibliographie. Sowerby. 'Thes. Conch. I. pag. 57. Pl. 13, fig. 14.

G. O. SARs. 1. c. pag. 2อ. Pl. 2, fig. 4. a-c.

Dragages. 1880. III, IV, V, VI, VIII, IX, X, XI, XII et XIII; les dragages XI et XII ont apporté 21 , les autres 18 exemplaires.

1881. Drag. X, 30 exemplaires; XI, 1 exemplaire; XVII, 6 exemplaires; XIX, 6 exemplaires. Synonymie. Pecten vitreus Grax (non Chemnitz).

1) Les dimensions que je donnerai dans la suite ont toujours rapport à l'exemplaire de la plus grande taille Nous ferons usage des abbréviations: $\mathrm{A}=$ Altitudo $=$ Hauteur $; \mathrm{L}=$ longitudo $=$ Longueur $; \mathrm{La}=$ Latitudo $=$ Largeur; toujours en millimètres. 
Distribution goégraphique. Les côtes septentrionales de la Scandinavie; Novaja-Semlja, Spitsbergen, Islande, Groenland et les côtes orientales de l'Amérique du Nord.

SARS a trouvé cette espèce depuis 30 jusqu'à 100 brasses de profondeur.

\section{Pecten islandicus, MüLL.}

$$
\mathrm{L}=62, \mathrm{~A}=66, \mathrm{La}=15 .
$$

Bibliographie. MülLer. Zoöl. Dan. Prodr. N. 2990.

Chemnitz. Conchyl. VII. pag. 314. Pl. 65, fig. 615, 616.

SARS, l. c. pag. 16. Pl. 2, fig. 2.

Govld, l. c. pag. 198, fig. 495.

Dragages. 1880. I, II et XIII. Des dragages I et. II ont été rapportés 9 exempl., du drag. XIII un seul exemplaire.

1881. IX, 1 exempl. avec 6 Anomia ephippium; XI, 9 exemplaires:

Synonymie. Ostrea is landica, MüLler.

Ostrea cinnabarin a, BörN.

Pecten Pealii, Conrad.

Pecten rubidus, Martrn.

Chlamys islandicus, Chenu.

Distribution géographique. Les côtes septentrionales (Finnmarken) de la Norvège, les Lofodes, les côtes méridionales et occidentales de la Norvège jusqu’à Bergen, Spitzbergen, le Groenland, l'Islande, la mer de Barents, les côtes orientales de l'Amérique du Nord.

\section{Fam. Limidae.}

\section{Lima elliptica, Jefreys.}

$$
\mathrm{L}=7,5, \mathrm{~A}=12, \mathrm{La}=8 .
$$

Bibliographie. Jeffreys, l. c. II. pag. 81 et V Suppl. Pl. XXV, fig. 2.

SARs, l. c. pag. 25.

Dragages. 1881. I, 2 exemplaires; II, beaucoup d'exemplaires.

Synonymie. L. subauriculata, Forb. et Hancock.

L. subauriculata, Mon'iag.

Limatula elliptica, JEFrRexs par SARs, l. c. page 25.

- Cette espèce a été recueillie depuis 15 jusqu'a 300 brasses de profondeur.

Distribution géographique. Les côtes occidentales de l'Ecosse, les îles Shetland, les côtes septentrionales et occidentales de la Scandinavie, la mer de Barents.

\section{Fam. Mytilidae.}

\section{Mytilus edulis ${ }_{9} \mathrm{~L}$.}

Bibliographie. Linné. Syst. nat. pag. 1157.

Forb. et Hancock. II. pag. 170. Pl. XLVIII, fig. $1-4$ et Pl. Q, fig. 5.

Dragages. 1881. XX à la profondeur de 15 brasses. 
WALter a recueilli cette espèce dans le Baffinsbay à une profondeur de 140 brasses.

Distribution géographique. Dans l'Europe du Groënland jusqu'a la Mediterranée; les côtes orientales et occidentales de l'Amérique du Nord, la mer de Behring et du Japon.

Cette espèce a $5-6$ dents bien accentuées à l'umbo, ensuite viennent $2-3$ plus petites qui maintefois ne font qu'une et alors encore deux qui sont de petite taille.

\section{Mytilus pellucidus, Pennant.}

$$
\mathrm{L}=33 . \quad \mathrm{A}=14 .
$$

Bibliographie. Pennant. British. Zoöl. IV. pag. 237. Pl. 66, fig. 3.

Wood. Linn. Transactions VI. Pl. XVIII, fig. 13 et 14.

Gould. 1. c. pag. 185.

Dragages. 1881. XI. Une coquille. Dans le port de Hammerfest 7 exemplaires.

Distribution géographique. Probablement la même que de M. edulis. Woop. Pl. XVIII, fig. 13 et 14 dessine les dents d'une manière peu exacte. Il en compte 3-7. Gould l. c. pag. 185 regarde les bandes comme le caractère le plus distinctif et le plus constant. D'après mon opinion les 2 ou 3 dents sont le caractère le plus distinctif. De plus les lamelles en arcade qui unissent les dents sont plus fortement accusées chez le M. edulis que chez M. pellucidus; ici elles sont très difficiles à remarquer ou manquent. Encore je trouve l'umbo très proéminent. C'est pourquoi avec Pennant et Furton je considère M. pellucidus comme une espèce et non comme une variété ainsi que le fait JEFFREYs, l. c. II. pag. 105.

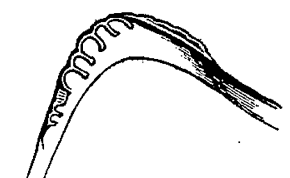

Les dents de M. edulis.

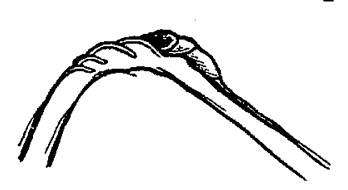

Les dents de M. pellucidus.

\section{Modiolaria nigra, Gray.}

$$
\mathrm{L}=37 . \mathrm{A}=20 . \mathrm{La}=11,5 \text {. }
$$

Bibliographie. Gray. Suppl. to App. to Parry's firs Voyage to the Northpole pag. 244.

JEFrReys. II. pag. 128 et V, Pl. XXVIII, fig. 4.

SARs, l. c. pag. 31 .

Gound, l. c. page 190. Fig. 488.

Dragages. 1880 . IX et $\mathrm{X} .8$ Exemplaires.

1881. XV et XVI. 10 coquilles.

Synonymie. Modiola nigra, Gray. l. c.

Mod. dis crepans, Flem. (non Lam.).

Modiola compressa, Menke.

Modiala nexa, Gould. 1ere Edition.

Modiola depressa, Hanley. Rec. Shells. I, 242 note.

Crenella nigra, Forb. et Hanc. II. pag. 202. Pl. XLIV, fig. 5 et Táb. Q, fig. 7.

Mytilus discors, Chemnitz.

Myt. discors var. Suecieus, O. Fabricius. Danske vidensk. 1780, pag. 460.

Myt. discrepans, Montagu.

Myt. pectinulus, Stimpson. Shells of New-Engl. II. (1851).

Modiolaria nigra, Loven.

Modiolaria striatula, Beck in Gaim. Voy. in Isl. Tab. 17. Fig. O et F. 
Distribution géographique. Les côtes de Yorkshire, de Northumberland et de Durham, de l'Ecosse et des Iles Shetland, Doggersbank, Kiel, les côtes du Danemarc et de la Scandinavie; Novaja-Semlja, l’Islande, le Groënland; les côtes de New-Foundland, de New-England et de Massachusets; à une profondeur de 7 jusqu' à 100 brasses.

\section{Modiolaria discors, $\mathrm{L}$.}

$$
\mathrm{L}=43 . \mathrm{A}=27 . \mathrm{La}=20 \text {. }
$$

Bibliographie. LInn. Syst. Nat. pag. 1159. Editio XII.

Forb. et Hanc. II. pag. 195. Pl. XLV, fig. 5 et 6 et Pl. XLVIII, fig. 5.

JeFFreYs, l. c. II. pag. 126 et V. Pl. XXVIII, fig. 3.

SARS, l. c. pag. 129 .

Gould, l. c. pag. 192, fig. 489 .

Dragages. 1881. XI. 1 exemplaire. V et XVIII, plusieurs exemplaires.

Synonymie. Mytilus discrepans, Monragu.

Myt. discors, L. et Simpson.

Modiola discrepans, LaM.

Modiola laevigata (var.?), Gray.

Modiolariadiscors, Loven. (Index Moll. Scand. 33).

Crenella discors, Forb. et Hancock, l. c.

JefrReYs. II. pag. 127 identifie Modiolaria la evigata et M. substriata, GraY. avec M. dis cors; cependant SARs la signale comme une espèce bien determinée.

Distribution géographique. Cette espèce habite communément avec la précédente, mais on la trouve aussi dans la Mediterranée et jusqu'au Japon.

Elle a été signalée à une profondeur de 4 jusqu' à 500 brasses.

Fam. Arcidae.

\section{Arca pectunculoides, Scacchr.}

$$
\mathrm{L}=8 .
$$

Bibliographie. Scacchi. Ann. Civ. d. Sicil. VI. pag. 82.

JefFreYs, l. c. II. 171.

Forb. et Hanc. II. pag. 241. Pl. XLV, fig. 8.

Saks, 1. c. pag. 43.

Dragages. '1881. I. Un exemplaire. $\mathrm{L}=6, \mathrm{~A}=4$; II, 2 exempl.

Synonymie. Arca raridentata,. Woon.

A. pusilla, Nyst.

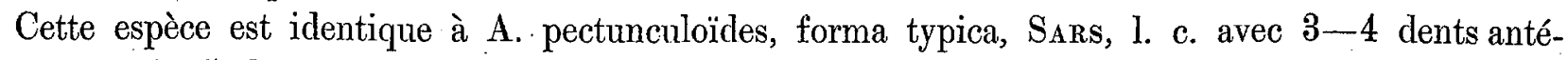
rieures et $4-5$ dents postérieures.

Distribution géographique. Les Hébrides et les Iles de Shetland, les côtes septentrionales et orientales de la Scandinavie, le Groënland, la mer de Barents, la Mediterranée. Elle a été recueillie depuis 60 jusqu’à 300 brasses de profondeur. 


\section{Arca septentrionalis, SARs.}

$$
\mathrm{L}=17 . \mathrm{A}=11 . \mathrm{La}=7,5 \text {. }
$$

Bibliographie. SARs, 1. c. pag. \$3. PI. IV, fig. 2, a-c.

Dragages. 1880. I, II, VIII, IX, X. Ont été recueillis sur les stations I et II 3 exemplaires; 4 exempl. dans le dragage VIII et sur les stations IX et $\mathrm{X}$ chaque fois un seul exemplaire.

1881. XX. A peu près 300 exemplaires.

M. le Prof. SARs donne la diagnose de cette espèce sous le nom de Arca pectunculoïdes var. septentrionalis", et dit qu'il est incliné à considérer cette bivalve comme une variation boréale, 》uden met sikkerhed specifiskt at kunne skilles fra hin (forma typica), dog maa betragtes som en klimatisk varietet.«

La grande quantité d'exemplaires bien conservés qui était à ma disposition m'a donné lieu d'entreprendre une diagnose exacte qui concorde avec celle du célèbre naturaliste de la Norvége. Elle a toujours $5-6$ dents antérieures et $7-8$ dents postérieures et une forme particulière bien caractéristique; c'est pourquoi je l'ai separée spécifiquement du Arca pectunculoïdes Scacchr.

\section{Arca glacialis, Gray.}

Bibliographie. GraY. Suppl. to the App. to Parry's First Voyage. On trouve une diagnose exacte par SARS l. c. pag. 43.

Dragages. 1881. XX. Deux valives.

Synonymie. Arca raridentata, var. maj. M. SARs ex parte.

Distribution géographique. Les côtes septentrionales de la Scandinavie, la mer de Barents.

Elle a été trouvé à une profondeur depuis 60 jusqu'à 170 brasses.

\section{Limopsis minuta, PhIL.}

$$
\mathrm{L}=9 . \mathrm{A}=8 . \mathrm{La}=4 \text {. }
$$

Biôliographie. Phmrppr. Fauna Moll. Sic. II.

SARs, l. c. pag. 44. Pl. III, fig. 5, a-c.

Jefreers, l. c. Suppl. V. pag. 174.

Dragages. 1881. VII. Un seul exemplaire.

Synonymie. Pectunculus minutus, Philippi l. c.

Limopsis borealis, Woodward.

Distribution géographique. Les Hébrides, les côtes septentrionales et orientales de la Scandinavie, les îles Shetland, la Méditerranée.

Elle a été trouvée à une profondeur de $70-400$ brasses. L'expédition du Talisman a recueillie cette espèce à une plus grande profondeur jusqu'au Senégal et vraisemblablement elle se trouverait jusqu'au Cap de Bonne Espérance. 
Fam. Nuculidae.

\section{Nucula tenuis, Montagu.}

$$
\mathrm{L}=13 . \quad \mathrm{A}=12 . \mathrm{La}=6,5 .
$$

Bibliographie. Montagu. Fest. Brit. Suppl. pag. 56. Pl. 29. Fig. 1.

GovLD, l. c. pag. 149.

FonB. et H., l. c. II. pag. 223. Pl. XLVII, fig. 6 et Pl. P, fig. 5.

JEFFREYS, l. c. II. pag. 151.

SARs, l. c. pag. 38 .

Dragages. 1880. IX et X; 3 exemplaires.

1881. IX 16 exemplaires; XII, 2 valves, XVII 15 exemplaires.

Synonymie. A rca tenuis, Mos'r. l. c.

$\mathrm{Nucula}$ tenuis, Furton et autores.

$\mathrm{Nucula}$ expansa, Mörch and ReEve. J Jefreeys identifie ce bivalve à N. tenui s ; peutêtre il est la variété »expansa《 de SARs.

Gould l. c. décrit le $\mathrm{N}$. tenuis avec »about eight teeth before and four or five behind the beaks.« Dans la diagnose de JEFFrEYs sont décrites 6 dents antérieures et 16 dents postérieures, tandis que SARS attribue 10 dents antérieures et 18 dents postérieures à cette espèce.

$\mathrm{Nucula}$ inflata, $\mathrm{H}_{\mathrm{ANCOCK}}=\mathrm{N}$. tenuis, MöLLER $=\mathrm{Nucula}$ obliquata, Beck d'après Goudo l. c. 153 aurait 5 dents antérieures et 10 dents postérieures.

$\mathrm{Nucula}$ expansa, Reeve $=\mathrm{N}$. Bellotii, Adams. aurait $10 \mathrm{~d}$. a. et $15 \mathrm{~d}$. postérieures.

Sars croit pouvoir distinguer $\mathrm{Nucula}$ inflata, H. = N. expansa, R. comme une simple variété.

Il me semble que le nombre des dents antérieures et postérieures est très variable comme il suit des 18 exemplaires, chez lesquels je les ai comptées.

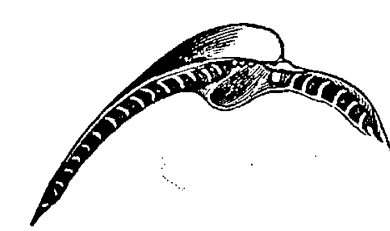

Les dents de Nucula tenuis.

$$
\begin{aligned}
& 6-14 ; 7-15 ; 8-18 ; 9-16 \text {; } \\
& 6-16 ; 7-16 ; 8-18 ; 9-18 \text {; } \\
& 6-17 ; 7-17 ; 8-18 ; 9-18 \text {; } \\
& 6-17 ; 7-17 ; 8-19 ; 9-20 \text {; } \\
& \text { 6-17; } 8-20 \text {; }
\end{aligned}
$$

Distribution géographique. Les côtes septentrionales et orientales de l'Angleterre et de l'Ecosse; les Hébrides et les îles Shetland. Doggersbank; les côtes du Danemark; les côtes septentr. et occid. de la Scandinavie; l'Islande, le Groënland, Spitzbergen, la mer de Barents; les côtes occidentales et orientales de l'Amérique du Nord, les côtes du Nord-Ouest de la France et de l'Espagne; la Mediterranée.

Elle a été trouvée depuis 25 jusqu' à 300 brasses de profondeur.

\section{Fam. Ledidae.}

\section{Leda pernula, MüLx.}

$$
\mathrm{L}=34 . \mathrm{A}=16 . \mathrm{La}=8 .
$$

Bibliographie. MüurER. Beschr. Berl. Nat. Fr. IV, pag. 57. Ed. 1779.

SARs 1. c. pag. 35. Pl. 5, fig. 1. a-d. 
Dragages. 1880. IV, V, VI, XIII et XIII A. 11 exemplaires sur ces quatre stations. 1880. XI et XII. 3 exemplaires.

1881. IX. 3 exemplaires; XIV 5 valves; XVII, 31 exemplaires.

Synonymie. Nucula oblonga, Brown.

Nucula cuspidata, Philippi.

Nuculana pernu.la, MüLLer l. c.

Distribution géographique. Les îles Shetland; les côtes septentr. de l'Angleterre; les côtes du Nord-Ouest de la Scandinavie; les côtes du Danemark; l'Islande, Spitzbergen, le Groënland, la mer de Barents, les côtes septentrionales et orientales de l'Amérique du Nord.

L'espèce a été recueillie depuis 15 jusqu'à 150 brasses de profondeur. Circumpolaire.

15. Leda minuta, MüLL:

$$
\mathrm{L}=13,5 . \quad \mathrm{A}=6,5 . \mathrm{La}=4 .
$$

Bibliographie. Müller. Prodr. Zoöl. Dan. page 247. No. 2985.

Forb. et Hanc. II. page 226. Pl. XLVII, fig. $11-13$ et Pl. P. fig. 2.

Gould 1. c. pag. 164.

Jefremeys l. c. II, pag. 155 et V Suppl. Pl. XXIX, fig. 6 .

Dragages. 1880. XI et XII.

Synonymie. Ar ca minuta, MüLL. l. c.

Arca minuta, Fabr. Fauna Gronl. pag. 414.

Le d a caudata, Donovan.

Le da c a udata, Forb. et Hancock l. c. et Lóven. Ind. Moll. Sc. p. 34.

Leda complanata, MöLt.

Nucula rostrata, Sowerby et Mc. Gilurvray.

Nucula minuta, TurTon.

Gould 1. c. distingue deux espèces, L. minuta et L. caudata; et il décrit aux deux espèces 12 dents antérieures et 14 dents postérieures. JEFFREXs en compte 16 et 20 . SARS dans sa diagnose dit, que comparée à L. pernula elle a moins de dents »denticulis cardinalibus paucioribus.« Je duute q'il y ait lieu de séparer spécifiquement L. minuta et L. caudata Don. comme le fait GoLd. Au contraire avec SARS il me semble nécessaire de réunir ces deux espèces. Quant à mes exemplaires, j'ai toujours trouvé 10 dents antérieures et 12 dents postérieures.

Distribution géographique. Les côtes de l'Angleterre, d'Irlande, de la France, de la Scandinavie, du Groënland, de l'Islande, de Spitzbergen et du Japon.

Cette espèce a été recueillie à une profondeur de 10 jusqu’à 150 brasses.

\section{Yoldia limatula, SAY.}

$$
\mathrm{L}=46 . \mathrm{A}=23 .
$$

Bibliographie. SAY. Americ. Conch. Pl. 12 (1831).

GovLD 1. c. pag. 155 .

SARs l. c. pag. 40. Pl. 4, fig. 12 ab.

Dragages. 1880. XIII, 3 exemplaires.

1881. IX, 15 exemplaires; XIV, une coquille. XVII, Plusieurs exempl. 
Synonymie. Nucula limatula, Say l. c.; Sow. Thes. Conch. Pl. I, fig. 9.

- Yoldia limatula, Adams et autores.

Leda limatula, Strmpson.

Yoldia arctica, MöLL.

Le nombre des dents me semble très variable. Gould decrits 22 dents antérieures et $18 \mathrm{~d}$. p. SARs 》usque ad 36 d. a et usque ad 29 d. post.« J'ai trouvé presque constamment $22 \mathrm{~d}$. ant. et $21 \mathrm{~d}$. postérieures.

Distribution géograplique. Les côtes orientales de l'Amérique du Nord. I'Islande; les côtes septentrionales et occidentales de la Scandinavie; la mer de Barents.

Cette espèce se trouvé ordinairement à une profondeur de 40 jusqu’à 200 brasses,

\section{Yoldia sapotilla, GouLb.}

$$
\mathrm{L}=14 . \mathrm{A}=7,5 . \mathrm{La}=6,5 .
$$

Bibliographie. Gound. Inv. Mass. page 100. Fig. 61. Ed. 1841.

Gould. Report etc. Page 159, fig. 466.

Dragages. 1880. IV, V, VI, XIII et XIII A. 9 exemplaires. XI et XII sur chaque station un seul exempl.

1881. X. Un seul exemplaire.

Synonymie. Nucula sapotilla, Gould.

Led a s a p otilla, STrmpson.

Yoldia sapotilla, Packard et autores.

Distribution géographlique. Les côtes septentrionales et orientales de l'Amérique du Nord; la mer de Barents.

\section{Fam. Cardiidae.}

\section{Cardium ciliatum, FABr.}

$$
\mathrm{L}=48 . \mathrm{A}=47 . \mathrm{La}=30 \text {. }
$$

Bibliographie. Fabricius. Fauna Groenl. page 410 (1780).

Möller. Moll. Groenl. page 20.

Linnaeus. Syst. Nat. page 1124.

Goold. 1. c. page 139 (Cardium islandicum).

SARs. l. c. page $46, \mathrm{Pl}$. 5, fig. 4, a-b.

Dragages. 1880. IV, IX, X, XIII; 10 exemplaires.

1881. XI, 2 exemplaires; XIV, 8 valves; XV, 15 valves; XVII, 3 exemplaires.

Synonymie. Cardium is landicum, L. et Crementz.

C. arcticum, Sowerby. Conch. Tll. fig. 26. 1841.

C. pubescens, Courhour. Boston Journ. Nat. Hist. II. Pl. 3, fig. 6.

Distribution géographique. Les côtes sept. et orient. de la Scandinavie, Spitzbergen, l'Islande.

le Groënland, Novaja-Semlja, la mer de Barents, les côtes orientales de l'Amérique du Nord;

Cette espèce a été trouvée sur les côtes de la Scandinavie à une profondeur de 10 à 20 brasses ; au large elle a été recueillie depuis 150 jusqu'à 200 brasses de profondeur. 
Fam. Cyprinidae.

\section{Cyprina islandica, $\mathrm{L}$.}

$\mathrm{L}=75 . \dot{\mathrm{A}}=70 . \mathrm{La}=45$.

Bibliographie. Linn. Syst. Nat. 1131.

Gould, l. c. page 129.

SARs. 1. c. page 50.

JEFrReYs. l. c. page 304 et $\mathrm{V}$ suppl. Pl. XXXVI, fig. 2.

Dragages. 1881. XIV, 4 exempl.; XV, une grande quantité.

Synonymie. Pectunculus maximus, Lister. Conch. Pl. 272, fig. 108.

Pectunculus crassus, Da Costa. Brit. Conch. page 183. Pl. 14, fig. 5.

Venus islandica, Linn. l. c.

Venus mercenaria, Pennant. Brit. Zoöl. IV. page 94. Pl. 53, fig. 47.

Venus bicardium, Born. Mus. Pl. 4, fig. 11.

Cyprina islandica, Lamarck. Anim. sans. Vert. II. Ed. Tome VI, page 290.

Cyprina vulgaris, Sowerby. Genera. Pl. 38, fig. 11.

Arctica vulgaris, Schum. Nouv. Syst. page 145. Pl. 13, fig. 13.

Cyprina arctica, Bowd. Elem. Conch. page 11, fig. 33.

Distribution géographique. Les côtes sept. et orient. de la Scandinavie et de la Russie, l'Islande,

le Groënland et les côtes orientales de l'Amérique du Nord; la mer de Barents.

Elle a été trouvée à une profondeur de jusqu’à 50 brasses.

Fam. Astartidae.

\section{Tridonta borealis, Снемк.}

$$
\mathrm{L}=46 . \mathrm{A}=42 . \mathrm{La}=24 .
$$

Bibliographie. Chemn. Conch. VII. Tab. 39, fig. 412.

GoulD. 1. c. page 121. Astarte semi-sulcata.

SARs. l. c. page 50. Pl. 5, fig. 8 , a-b.

JEFFREYs. l. c. II. page 320 et $\mathrm{V}$ suppl. page 183.

Dragages. 1881. IX, 31 exempl.; Busse-sund près de Vardo, 1 exempl.; XI, 1 exempl. et une valve; XIV, 2 exempl.; XV, 2 coquilles.

Synonymie. Venus borealis, Chemn. 1. c.

Astarte borealis, Philrppr.

A. arctica, Forb. et Hancock. Brit. Moll. I. page 461. Pl. 30, fig. 7.

A. semi-sulcata, Grax. App. t. Parry's Voyage.

Crassina semi-sulcata, Leach in Ross Voyage. App. 175.

Cr. borealis, Nuxuson. Nova acta Holm. page 188. Pl. II, fig. 3 et 4.

Astarte cyprinoüdes, Duval. Rev. Zoöl. page 278 (1841).

Venus compressa, Montagu. Test. Brit. Suppl. Pl. 26, fig. 1.

Astarte compressa, Mc Gillivray. Moll. Aberd. page 261.

Crassina compressa, Brown. Ill. Conch. Gr. Britt. p. 96. Tab. 38, fig. 4 et 5.

$\gg$ corrugata, $\gg$ Idem. Tab. 40, fig. 24 . 
Astarte corruga, Loven. Ind. Moll. Scand. page 37.

$\gg \quad$ lactea, Gould. Inv. Mass. 1e éd. page 80, fig. 47.

Distribution géographique. Les côtes septentrionales de la Russie et de la Scandinavie; Spitzbergen, Groënland; les côtes orient. de l'Amérique du Nord. La mer de Barents.

Cette espèce a été recueillie depuis 20 jusqu’à 200 brasses de profondeur.

\section{Astarte sulcata, DA Costa.}

$$
\mathrm{L}=28 . \mathrm{A}=23 . \mathrm{La}=12 \text {. }
$$

Bibliograplie. DA Cost'A. Brit. Conch. page 192. Ed. 1778.

JeFrreYs. 1. c. II. page 313 et V. PI. XXXVII, fig. 1.

Gould. l. c. page 119, fig. 432 .

SARs. 1. c. page 52 .

Dragages. 1880. Sur toutes les stations.

1881. VII, une paire de coquilles; IX, 3 exemplaires; X, un seul exemplaire; XVIII, un seul exemplaire; XX, 8 exemplaires.

Synonymie. Pectunculus sulcatus, DA Cost'A. l. c.

Venus sulcata, Montagu. Test, Brit. page 131 .

Astarte sulcata, Fuem. Brit. Anim. page 439.

Venus'Damnonia, Mon'. Test. Brit. Suppl. page 45. Pl. 29, fig. 4.

Venus Damnoniensis, Bliainville.

Crassina sulcata, TurTon.

Cr. Damnoniensis, Lam.

Astarte Damnoniensis, Sow.

Venus scotica, Mont. Test. Brit. Suppl. 44.

Astarte scotica, Flem. Br. An. page 44.

Crassina scotica, Leach in Pross. Voy. page 175.

Distribution géographique. Les côtes sept. et orient. de l'Amérique du Nord; les côtes du Groënland, de l'Islande et de Spitzbergen; la mer de Barents, Novaja Semlja; les côtes septentrionales de la Russie et de la Scandinavie, les côtes occidentales de la Scandinavie et du Danemark.

\section{Astarte elliptica, Brown.}

$$
\mathrm{L}=29 . \mathrm{A}=21 . \mathrm{La}=11,5 .
$$

Bibliographie. Brown. Edinb. Nat. et Geol. Sc. I. page 12. Pl. 1, fig. 8 et 9.

Govm. l. c. page 124, fig. 435 .

SARs. l. c. page 53.

JEFFREYs. l. c. II. page 312.

Dragages. 1880. Sur toutes les stations.

1881. II, 2 exemplaires; XV, 4 valves; XVIII, 6 exemplaires; XX, 8 exemplaires.

Synonymie. Crassina elliptica-ovata, Brown. 1. c.

Crassina sulcata, Nillson. Nova acta Holm. 187. Pl. 2, fig. 1 et 2.

Cr. elliptica, Hanley. Rec. Shells. Suppl. Pl. 14, fig. 36. 
Astarte elliptica, Mc Gilurvray. Moll. Aberdeen. page 259, et Forb, et Hanc. Br. Moll. I. 459 .

Astarte semi-sulcata, MöLl. Ind. Moll. Groenl. page 19 (non Leach).

Venus compressa, L.

Distribution géographique. On la trouve vraisemblablement en compagnie de la précédente à une profondeur de 7 à 300 brasses.

Je crois avec Sars et contra Jefrrers que l'Astarte elliptica doit être separé specifiquement de l'Astarte sulcata. D'après Jefrekrs l'Ast. elliptica serait une variété boréale de l'Ast. sulcata.

La circonstance qu'elle a été trourée dans l'été de 1880 en compagnie de l'Ast. sulcata sur toutes les stations ne donne pas beaucoup de valeur à l'opinion citée.

Il me semble trés douteux que l'Astarte crebricostata Forbes serait une espèce bien destincte, pourtant je la signalerai comme telle sous le $\mathrm{n}^{0}$. suivant.

\section{Astarte crebricostata, Fonbes.}

$$
\mathrm{L}=19 . \mathrm{A}=16 . \mathrm{La}=7,5 .
$$

Bibliograplie. Fonbes. Ann. Nat. Hist. XIX, pag. 98. Pl. 9, fig. 4.

Gould, l. c. pag. 127.

SARs, l. c. pag. 54. Pl. 5, fig. 7 a-b.

Dragages. 1881. IX, 11 exemplaires. XVIIT, 8 exemplaires.

Synonymie. A. depressa, Brown. (?)

A. sulcata var. multicostata, JeFrrexs. (?)

Distribution géograplique. Comme l'Astarte sulcata.

24. Nicania Banksii, Leach.

$$
\mathrm{L}=18 . \mathrm{A}=15 . \mathrm{La}=8 \text {. }
$$

Bibliograplie. Leach on Ross Voy. App.

Gray. Zoöl. Beechey's Voy. Pl. 44, fig. 10.

Sowerby. Thes. Conch. II, page 782. Pl. 167, fig. 8.

Gould. I. c. page 125, fig. 438.

SARS. 1. c. page 51 .

Dragages. 1881. IX, 28 exemplaires. XIV, 4 exemplaires. XV, un seul exemplaire.

Synonymie. Astarte Banksii, Leach l. c.

Venus compressa, Montagu.

Cyprina Montagui, Risso.

Astarte pulchella, Jonas.

A. propinqua, Landsborovgh.

Venus Montacuti, Turmon.

Crassina convexiuscula, Brown.

C. obliqua, Brown.

Astarte multicostata, J. SмIтH.

A. Ud devaliensis, J. Smith 
Distribution géographique. Les côtes septentrionales de la Russie et de la Scandinavie, Spitzbergen, le Groënland, la mer de Barents, les côtes septentrionales et orientales de l'Amérique du Nord.

L'espèce à été trouvée à une profondeur de 10 à 140 brasses.

Fam. Tellinidae.

25. Tellina tenera, LEACH.

$$
\mathrm{L}=35 . \mathrm{A}=25 \text {. }
$$

Bibliographie. Leach. Appendix to Rose's Voyage I, page 175.

SARs. 1. c. page 76. (M a c o m a c a l c a ri a Chennitz.)

Gould. 1. c. page 95 , fig. 401. ( $\mathrm{M}$ a c o m a p roxima.)

Dragages. 1880. IX, 1 exemplaire. X, 1 exemplaire.

1881. IX, En giande quantité. XII, 2 exemplaires. XIV, 10 valves. XV, 9 valves. XVII, 60 exemplaires.

Synonymie. Tellin a lata, GMelin et Lovén.

Ma coma calcaria, Chemnitz.

- Tellina calcaria, Lrell.

Tellin a sabula, Spengl. Mörch.

T. inconspicua, Broderip et Sowerby.

T. p roxima, Gray et Sмith.

M acoma proxima, Brown.

Ma croma tenera, Leach. l. c.

Tellina sordida, Conthour.

S anguinolaria sordida, Gound. Inv. M. I. Ed.

Distribution géographique. Le Groënland, la mer de Barents, et les côtes orientales de l'Amérique du Nord.

Cette espèce a été recueillie à une profondeur de 40 à 165 brasses.

Fam. Pandoridae.

\section{Lyonsia norvegica, Chemnitz.}

$$
\mathrm{L}=23 . \mathrm{A}=15 . \mathrm{La}=10 \text {. }
$$

Bibliographie. Chemnitz. Conch. XX, page 340. Pl. 170, fig. 1648, (M y a n o r ve gi c a). JeffreYs. 1. c. III, pag. 29 et V, Pl. XLVIII, fig. 2.

SARs. 1. c. page 81 .

Dragages. 1881. XVII, ว̊ exemplaires.

Synonymie. M y a nitida, Fabric. (non MüLtær).

M. striata, Montagu.

M. pellucida, Brown.

Amphidesma corbuloïdes, Lamarck.

Myatella Montagui, Brown.

Tellina coruscans, Scacchi.

Pandora a equivalvis, Phimrpr. 
Distribution géographique. Les côtes des îles britanniques, de la Scandinavie, la mer de Barents; les côtes de la France et de Madère; la Méditerranée.

Elle a été trouvée à une profondeur de $4-70$ brasses au Sud.

Fam. Saxicavidae.

27. Saxicava arctica, $\mathrm{L}$.

$\mathrm{L}=18 . \mathrm{A}=8,5 . \mathrm{La}=6,5$.

Bibliographie. Linn. Syst. Nat. Ed. XII, page 1113. (Mya arctica.)

Gould. 1. c. page 89.

JEFFRLYS. III, page 81 et V. Pl. XLI, fig. 4.

SARS. 1. c. page 95 . Pl. 20, fig. 8 , a-c.

Dragages. 1880. XIII A, 2 exemplaires. XI et XII, 2 valves. XV. plusieurs valves.

1881. XVII, 1 exemplaire. XXI, 6 exemplaires.

Synonymie. M y a arctica, Lins. l. c. paga 1113.

Solen minutes, Linn. l. c. paga 1115.

Mytilus praecicus, Mont.

Hiatella arctica, LAM.

Anatina arctica, Turton.

Hiatella minuta, Turron.

A gina purpurea, Turton.

Solen purpureus, Fuem.

Saxicava purpurea, Brown.

Saxicava rubra, Desh.

Saxicava rhomboïdes, Desh. , El. Conch.

Saxicava arctica, Desh. f Pl. 12, fig: 8 et 9 .

Rhomboïdes arctica, de Blarnv.

Distribution géographique. Comme la S. rugosa:

\section{Saxicava rugosa, Penn.}

$$
\mathrm{L}=34 . \mathrm{A}=17 . \mathrm{La}=14 \text {. }
$$

Bibliograplie. Pennant. Brit. Zö̈l. IV, pag. 110. Pl. 63, fig. 72.

Gould. 1. c. p. 87, fig. 397.

Sars. 1. c. p. 95 . Pl. 20 , fig. 7 , a-c (S. pholadis, L.).

JEFrReys. l. c. III, p. 81 et V. PI. LI Suppl., fig. 3.

Dragages. $1880 . \mathrm{XV}, 8$ valves.

1881. XVII, 5 exemplaires; XXI, 6 exemplaires.

Synonymie. Mytilus pholadis, L.

M. rugosus, Montagu.

Saxicava rugosa, LAM.

- pholadis, Lam.

distorta, SAY.

Mya bysifera, O. Fabr.

Byssomya pholadis, Bowditch.

Hiatella rugosa, Fuem. 
Distribution géographique. Groënland, Novaja-Semlja, Spitzbergen, la mer de Barents, l'Island; les côtes de la Scandinavie et du Danemark; les côtes septentrionales et orientales de l'Amérique du Nord; les côtes des îles Britanniques, les côtes occidentales de la France et de l'Espagne; la Méditerranée.

\section{Fam. Corbulidae.}

\section{Neaera arctica, M. SARs.}

$$
\mathrm{L}=21 . \mathrm{A}=15 . \mathrm{La}=11,5 .
$$

Bibliographie. SARs. 1. c. page 86. Pl. 6, fig. 5, a-c.

Dragages. 1880. IX et $\mathrm{X}$, un seul exemplaire.

1881. XX, un seul exemplaire.

Distribution géographique. Les côtes septentrionales de l’Amérique du Nord; les îles de Shetland, les côtes septentrionales de la Scandinavie, la mer de Barents.

Les draguages du Talisman ont trouvé cette espèce dans la golfe de Gascogne et jusqu'au Sahara.

\section{Mya truncata, L.}

Bibliographie. L. Syst. Nat. p. 1112.

GouLd. 1. c. pag. 59 , fig. 376 .

Jeffreys.. l. c. III, pag. 67 et V Suppl. Pl. L, fig. 2.

SARs. l. c. pag. 92.

Dragages. 1881. XV, Deux valves.

Synonymie. Mya priapus, Tumesius. Mém. de l’Acad. de Pétersbourg. VIII. p. 295. Pl. IX. -My a udevaliensis, Forb. et Hanc. Ann. et Mag. N. H. XVIII. p. 1846. Sphaenia $=$ Sphenia $\mathrm{S} w a i n s o n i$, Tur't.

Distribution géographique. Spitzbergen, l'Islande, le Groënland, la mer de Barents, les côtes sept. de l'Amérique du Nord; les îles Britanniques, les côtes occidentales de la France et de l'Espagne.

Cette espèce a été trouvé à une profondeur de 100 brasses dans les régions arctiques. 


\section{Tableau des Draguages effectués par le "Willem Barents"}

durant les mois de Juin-Juillet 1880 et de Juin jusqu'à Septembre 1881.

1880.

\begin{tabular}{|c|c|c|c|c|c|c|c|}
\hline \multirow{2}{*}{ ate. } & \multirow{2}{*}{$\begin{array}{l}\text { Numéro } \\
\text { d'ordre. }\end{array}$} & \multicolumn{2}{|c|}{ S i t u a t ion. } & \multirow{2}{*}{$\begin{array}{c}\text { Profon- } \\
\text { deur } \\
\text { enbrasses }\end{array}$} & \multicolumn{2}{|c|}{ Température de l'eau. } & \multirow{2}{*}{ Nature du fond. } \\
\hline & & Latitude. $\mathrm{N}$. & Longitude. E. & & au fond. & à la surface. & \\
\hline $22 / \mathrm{VI}$ & $\mathrm{I}$ & $74 \circ 30^{\prime}$ & $26^{\circ} \quad 3^{\prime}$ & 180 & $2,3^{\circ} \mathrm{C}$ & & Fond argileux et gros graviers. \\
\hline $23 / \mathrm{VI}$ & II & $74^{\circ} 36^{\prime}$ & $24^{\circ} 47^{\prime} 5^{\prime \prime}$ & 112 & $0,6^{\circ} \mathrm{C}$ & & $\begin{array}{l}\text { Fond rocheux avec uné couche } \\
\text { argileuse. }\end{array}$ \\
\hline $25 / \mathrm{VI}$ & III & $75^{\circ} \quad 3^{\prime} 5^{\prime \prime}$ & $27^{\circ} 12^{\prime}$ & 146 & $0^{\circ}, 0 \mathrm{C}$. & & Fond argileux avec des graviers. \\
\hline $29 / \mathrm{VI}$ & IV & $74^{\circ} 32^{\prime}$ & $35^{\circ} 50^{\prime}$ & 147 & $-1,4^{\circ} \mathrm{C}$ & & Fond argileux. \\
\hline $30 / \mathrm{VI}$ & $\mathrm{V}$ & $74^{\circ} 75^{\prime}$ & $34^{\circ} \quad 6^{\prime}$ & 175 . & $0,9^{\circ} \mathrm{C}$ & & La drague n'a pas été au fond. \\
\hline $1 / \mathrm{VII}$ & VI & $73^{\circ} 28^{\prime} 5^{\prime \prime}$ & $23^{\circ}$ & 160 & $1,8^{\circ} \mathrm{C}$ & & Fond argileux avec des graviers. \\
\hline $2 / \mathrm{VII}$ & VII & $72^{\circ} 41^{\prime}$ & $31^{\circ} 49^{\prime}$ & 136 & $1^{\circ} \mathrm{C}$. & & Vase noirâtre. \\
\hline $3 / \mathrm{VII}$ & VIII & $72^{\circ} 12^{\prime}$ & $31^{\circ} 50^{\prime}$ & 160 & $2,2 \cdot \mathrm{C}$ & & Vase noirâtre avec des graviers. \\
\hline $9 / \mathrm{VII}$ & IX & $70^{\circ} 80^{\prime} 5^{\prime \prime}$ & $34^{\circ} 11^{\prime} 7^{\prime \prime}$ & 140 & $1,4^{\circ} \mathrm{C}$ & & Fond argileux très compacte. \\
\hline 10/VII & $\mathrm{X}$ & $70^{\circ}, 9^{\prime}$ & $35^{\circ} 36^{\prime} 8^{\prime \prime}$ & 92. & $1,1^{\circ} \mathrm{C}$. & & Fond argileux avec des graviers. \\
\hline $12 / \mathrm{VII}$ & $\mathrm{XI}$ & $70^{\circ}, 48^{\prime}$ & $38^{\circ}$ & 115 & $1,1 \circ \mathrm{C}$ & & Vase noirâtre avec des graviers. \\
\hline $13 / \mathrm{VII}$ & $\mathrm{XII}$ & $71^{\circ} 18^{\prime}$ & $42^{\circ} \quad 41^{\prime}$ & 120 & $0,7^{\circ} \mathrm{C}$ & & Vase noirâtre avec des graviers. \\
\hline $14 / \mathrm{VII}$ & XII A & $71^{\circ} 8^{\prime} 2^{\prime \prime}$ & $44^{\circ} 25^{\prime} 7^{\prime \prime}$ & 80 & $1,1^{\circ} \mathrm{C}$ & & Fond argileux noirâtre. \\
\hline $23 / \mathrm{VII}$ & XIII & $75^{\circ} 14^{\prime} 2^{\prime \prime}$ & $44^{\circ} 26^{\prime} 4^{\prime \prime}$ & 130 & $-1,3^{\circ} \mathrm{C}$ & & Fond argileux avec des graviers. \\
\hline $26 / \mathrm{VII}$ & XIII A & $75^{\circ} 20^{\prime} 5^{\prime \prime}$ & $46^{\circ} 40^{\prime}$ & 150 & $-0,1^{\circ} \mathrm{C}$ & & Vase noirâtre. \\
\hline $30 / \mathrm{VII}$ & $|\mathrm{XIII} B|$ & $74^{\circ} 41^{\prime} 4^{\prime \prime}$ & $50^{\circ} 23^{\prime}$ & 84 & $\mid-0,6^{\circ} \mathrm{C}$ & & Vase noirâtre avec des graviers. \\
\hline
\end{tabular}




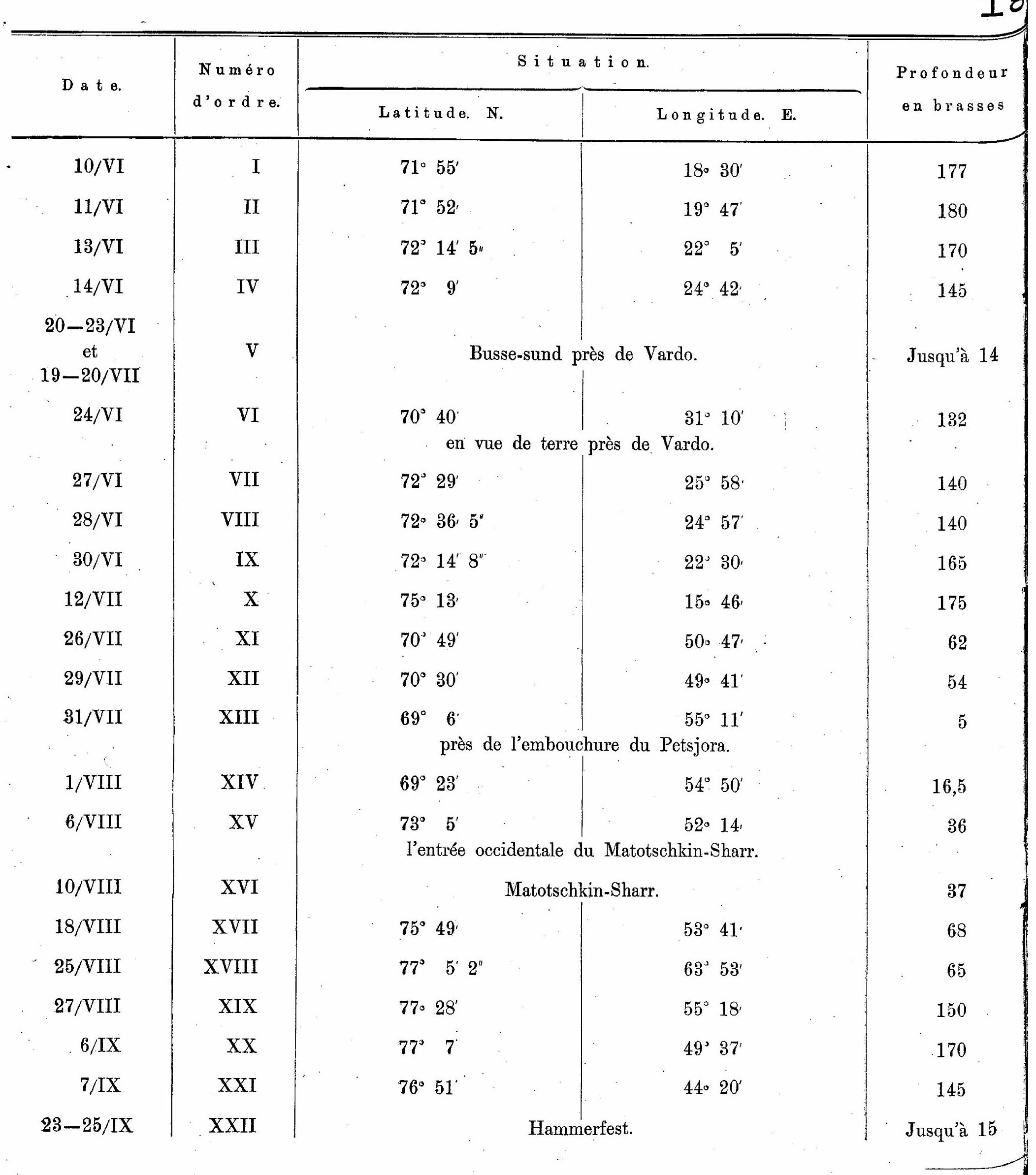




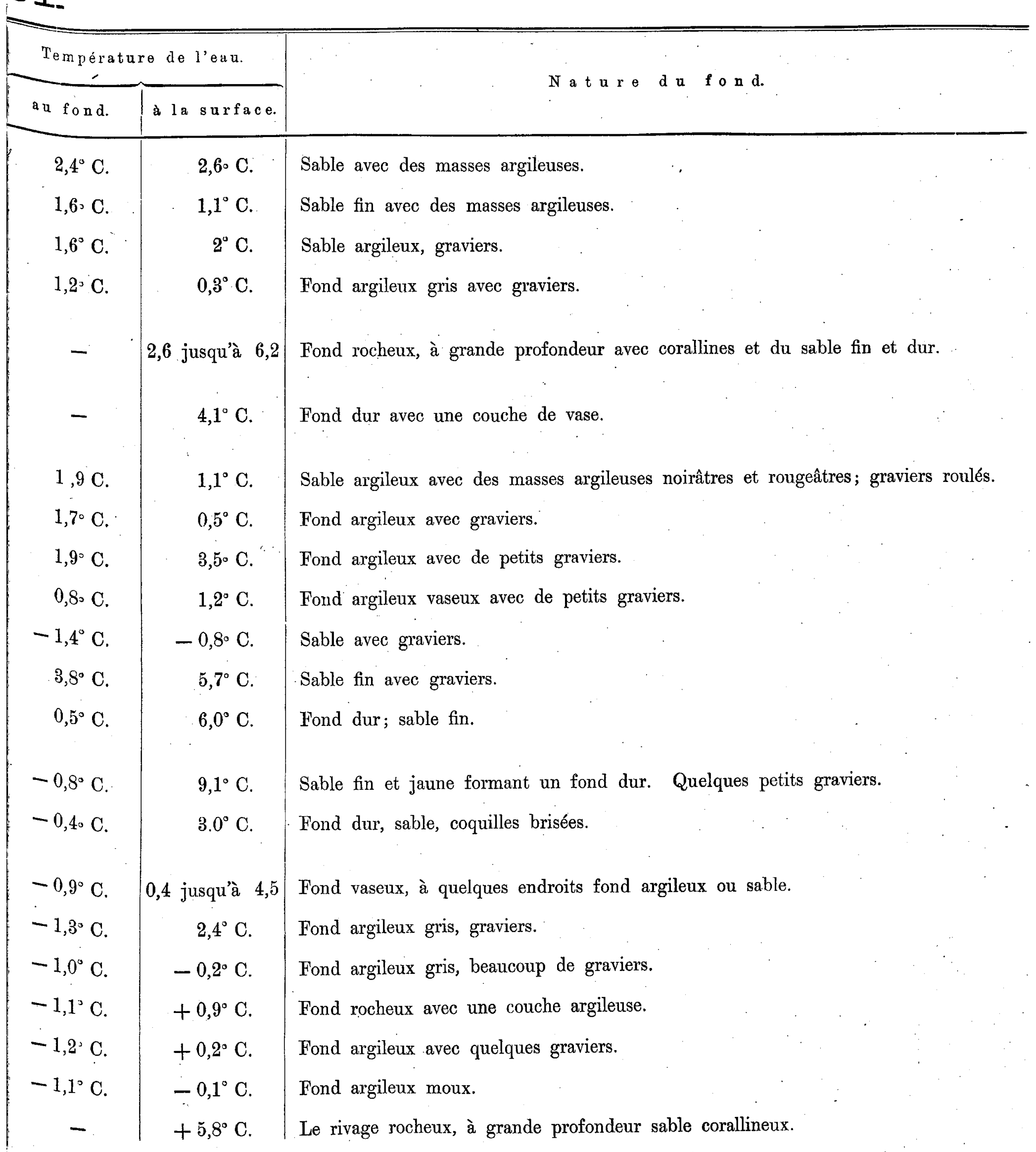




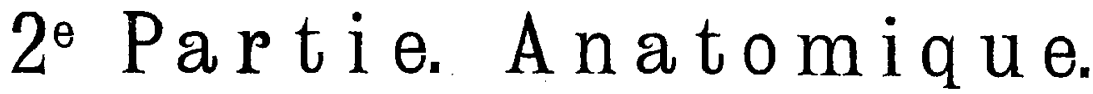

\section{A. Sur les organes byssogènes.}

Il paraît que ni Aristotète ni Puine ne font mention dans leurs écrits du byssus, qui se trouve chez quelques Lamellibranches. Et si nous passons Tertullien, Procope et Phile, dont les écrits ont été cités par $\mathrm{A}$. MüLLER, ce n'est que dans le $16^{\text {ième }}$ et 17 ième siècle que quelques naturalistes, Bellonius, Rondelet, Aldrovandi et Bochartus se sont occupés de la formation $\mathrm{du}$ byssus, sans toutefois se baser sur une dissection anatomique. A la fin du 17ième siècle parut un mémoire, excellent pour son temps, sur la moule commune par notre compatriote A. DE HeIDE, médecin à Middelbourg. L'auteur décrit le pied linguiforme (Spinnvinger. Carrière) et les muscles rétracteurs antérieurs qui vont s'insérer dans une fossette allongée en arrière de l'extrémité antérieure de la charnière. Du pied linguiforme il dit, qu'il est mobile et rétractile et qu'il prend des dimensions et des formes variées. De plus il a observé le sillon longitudinal à la face ventrale du pied et les glandes byssogènes, qu'il distingue comme. le pulpa. A l'extrémité libre du pied il a vu un orifice qu'il dit communiquer avec la cavité du système circulatoire, car par insection il a rempli les vaisseaux sanguins du foie, de l'ovaire etc. De plus il déorit les trois vaisseaux sanguins ( $\$ 27$ ), l'un médian, les autres latérals et en dessine une coupe transversale. Encore DE HEIDE a observé que le byssus est formé dans le sillon longitudinal et est attaché au tronc de byssus qui se trouve à l'entrée de la cavité byssifère. Plus loin (\$29) il donne une description de la manière dont la moule attache ses fils de byssus à un corps étranger. Bien que plusieurs de ses autres observations ne soient pas absolument correctes et que entre autres ses opinions sur la manière dont les fils de byssus croissent en épuisseur soient erroneuses, cet auteur est le premier qui par observation et par dissection ait étudié la formation du byssus.

Une trentaine d'années plus tard et comme il paraît indépendamment de notre cornpatriote, Réaumur a fait les mêmes observations que celui-ci sur la manière dont la moule s'attachait à divers objets plus ou moins éloignés. D'ailleurs il a fait plusieurs expériences avec des moules dépouillées de leurs fils. Il décrit la fente qui va de l'origine du pied linguiforme jusqu'au près de sa pointe et il a observé que ce canal finit dans un tuyau qui contient dans son milieu une espèce de tendon rond, ou plutôt un fil de même nature que les autres, mais beaucoup plus gros. C'est à ce tendon que sont attachés par une de leurs extrémités tous les fils déliés qui servent à fixer la moule. Il croit que la matière byssale est formée dans le sillon et que par des contractions musculaires, l'animal fait sortir la matière en dehors de l'orifice qui se trouve à la partie proximale du pied. D'après Réaumur le tronc de byssus - le tendon rond sortirait de l'orifice proximale comme les cheveux sortent de l'épiderme.

Nous ne nous arrêterons pas aux descriptions sommaires de quelques autres auteurs (D'ARGENvilue, Lesser, Chemnitz et autres) dont les écrits ne contiennent que des hypothèses ou les

1) A. MülLLer. Über die Byssus der Acephalen etc. Archiv für Naturgeschichte. III Jahrg. Bd. I. pag. 2. sqq

2) A. DE HEIDE. Ontledinge der Mossels en ontleed-, genees- en heelkundige waarnemingen. Uit het Latijn vertaald door F. J. van Almeloveren. Amsterdam, 1684.

3) Réaumur. Des différentes manières dont plusieurs espèces d'animaux de mer s'attachent etc. Histoire de l’Académie royale des sciences. Année 1711. pag. 108 sqq. 
opinions de leurs prédécesseurs, et nous passerons directement à PouI ${ }^{1}$ ). Cet auteur donne une description très correcte des plis ou cloisons de la cavité byssifère et comment le tronc du byssus se divise en une grande quantité de lamelles qui s'insèrent entre ces plis. De plus il croit devoir distinguer deux sortes de fils du byssus, ceux qui sont formés dans la cavité byssifère et dérivent $\mathrm{du}$ tronc; ces fils croissent comme des cheveux. Les autres fils sont formés par la matière muqueuse du pied linguiforme et servent à réparer les fils usés et à rattacher les fils du tronc de byssus à un corps étranger, si le byssus a été déchiré.

$\mathrm{Au}$ 'commencement de notre siècle Blainville ${ }^{2}$ ) décrit $\gg$ un plus ou moins grand nombre de »fibres des muscles adducteurs qui peuvent être attachées et s'agglutiner par leur extrémité élargie 》aux corps étrangers, de manière à servir de point d'appui extérieur à l'animal, c'est ce qui $\gg$ constitue le byssus dans les jambonneaux (Pinna), les moules (Mytilus) et le pied tendineux $\gg$ des tridacnes et de certaines espèces d'arches (Arca), etc., byssus qui n'est réellement pas »formé, comme quelques auteurs l'ont dit, d'une mucosité sécrétée par une glande et filée dans \une rainure du pied, mais qui n'est qu'un assemblage de fibres musculaires desséchées dans 》une partie de leur étendue, encore contractiles, vivantes à leur origine et qui même l'étaient »dans toute leur longueur a l'époque où elles ont été attachées «

Une douzaine d'années plus tard, A. MüLler ${ }^{3}$ ) publia sés recherches sur le byssus des Lamellibranches. Dans son mémoire il traite de la grosse anatomie des organes byssogènes de Tichogonia Chemnitzii, Rosm. (= Dreyssena polymorpha, van Ben.), Tridacna elongata, Lam., Malleus vulsatellus, Lar., Mytilus edulis, L., Myt. exustus, L., Pecten varius, LAm., Arca barbata, L., Lima squamosa, LAM., Lima glacialis, LAM., Meleagrina margaritifera, LAM., Perna sp. et Pinna nobilis, Pour. Quoique l'auteur ait plus spécialement étudié la structure du byssus des différentes espèces sus-nommées, il n'a pourtant pas négligé les glandes byssogènes et la grosse anatomie des pieds fileurs. Il décrit une glande byssogène (glandula byssipara) qui chez le Dreyssena polymorpha, Mytilus edulis et les autres espèces se trouve comme »einen weissen durchsichtigen Streifen zu beiden Seiten der Längsfurche.« Cette masse est de nature parenchymateuse et formée de acini. Cette glande fournit la matière byssale (Byssusmaterie). Il distingue en outre une matière agglutinative (Verbindungsmaterie) qui est sécrétée dans la cavité byssifère. MüLLER a cru trouver les orifices des glandes byssogènes et en décrit chez le Mytilus e duli s sept, qui sont situés à l'entrée du sillon longitudinal. Il n'a pu constater leur présence chez le Dreys sena, ni chez aucune des autres espèces mentionnées et ne fait done que présumer leur présence chez celles-ci. La matière agglutinative est sécrétée selon lui par une membrane qui tapisse la cavité byssifère et »vom Bauche kommend sich durch den Eingang zur Byssushöhle hineinschlägt « ${ }^{4}$ ).

A tout byssus MüLles distingue trois parties: une partie proximale, la racine »die Wurzel《, cachée dans la cavité byssifère et composée de lamelles ou de fibres; une partie médiane, le tronc »der Stamm《 et la partie distale, composée de fils, par lesquels l'animal s'attache aux corps étrangers. Ces fils sont formés d'après lui par la matière byssale, tandis que le tronc et la racine sont composés en partie de matière byssale et en partie de matière agglutinative. Cette matière agglutinative peut prendre part à la formation du tronc de byssus de deux manières; 10. en enveloppant la matière byssale qui donne naissance à des fils de byssus. Dans le second cas la matière agglutinative est enveloppeé par la matière byssale qui forme une écorce (Rinde). Alors chez la matière byssale »herrscht die Ausdehnung in der Fläche vor.« Résumant, nous obtiendrons la classification suivante:

1) Polr. Testacea utriusque Siciliae eorumque historia et anatome. Parmae. 1791-1827. II. pag. 195 et 196.

2) H. M. Ducrotay de Blainville. Manuel de malacologie et de conchyliologie, contenant etc. Paris. 1825. p. 115.

3) A. MÜller, l. c. pag. 12.

4) Mülter, 1. c. pag. 33. 
Le Byssus avec une écorce de matière byssale et agglutinative; la racine avec des lamelles.
Le Byssus sans cette
écorce.

$\begin{array}{cc}\text { Avec des fils à la partie } & \text { Sans fils à cette } \\ \text { distale. } & \text { partie. }\end{array}$

Dreyssena polymorpha.

Tridacna elongata.

Malleus vulsellatus.

Mytilus edulis.

M. exustus.

Pecten varius.

Arca barbata.

\author{
Lima squamosa. \\ L. glacialis. \\ Meleagrina margaritifera.
}
La racine avec des lamelles.
La racine sans lamelles.

Perna. .

Pinna nobilis.

Nous discuterons plus tard cette classification et son chapitre: $\gg$ Erklärung der Formen der Byssus« ou il donne une interprétation de la manière dont la matière byssale sécrétée dans le sillon longitudinal monte jusque dans la cavité byssifère, pour s'unir là d'une manière quelconque avec la sécrétion de cette cavité »la matière agglutinative«.

Longtemps les recherches de MüLLeR furent les seules en matière de byssus, lorsque Trcho Tullberg ${ }^{1}$ ) vint les détrôner. Ce naturaliste démontre, se basant sur des coupes transversales et longitudinales, avec une clarté sobre et minutieuse que le Byssus est formé chez la moule commune par des glandes qui toutes sont constituées de la même manière. Les acini de ces glandes se distribuent dans le pied et dans les flancs de la cavité byssifère; il n'a pu distinguer deux sortes de matières, qui prennent part à la composition du byssus, ni chez le Mytilus edulis, ni chez les autres espèces, qu'il a comparées, quant à la formation du byssus avec la moule commune.

Presque en même temps $\mathrm{SABATIER}^{2}$ ) en donnant une anatomie de la moule commune, décrit la présence d'un sillon ventral au pied linguiforme et d'une ouverture au commencement de ce: sillon, qu'il dit être »un orifice qui fait communiquer la cavité du système sanguin avec l'eau »au milieu de laquelle l'animal est plongé.«s) Du byssus ou des organes byssogènes il ne dit mot. Les auteurs qui récemment ont traité des glandes byssogènes sont Carrière et TH. Barrois. Dans différentes notes préliminaires et dans des mémoires - dont nous ferons mention en lieu convenable - ces naturalistes ont publié leurs recherches. Ils ont trouvé que chez les espèces qu'ils. ont examineés, les ouvertures, signalées par DeLLA ChIAJE ${ }^{4}$ ) comme »fori aquiferi« sont les ouvertures de glandes, qui sécrétant une matière granuleuse et agglutinative ont été nommées 》glandes byssogènes.« Contrairement au résultats de Kollmann et Griesbach que nous discuterons dans la dernière partie de ce mémoire, les naturalistes ci-dessus nommés émettent l'opinion que les pores aquifères de ces auteurs ne sont pas du tout les orifices d'un système aquifère et que: s'il y a lieu de parler de l'introduction de l'eau dans le système circulatoire des Lamellibranches, cette introduction n'a pas lieu au moyen d'un porus aquiferus dans le sens des auteurs.

Dans une notice préliminaire ${ }^{5}$ ) je me suis déjá déclaré comme ne partageant pas l'opinion de. Kollmann et de Griesbach. Je donnerai à present plus detaillés les résultats que j’ai obtenus. $\mathrm{Au}$ moyen de coupes tranversales et longitudinales j'ai étudié l'anatomie microscopique du pied des espèces suivantes, qui étaient durcies soit par l'alcool absolu, (celles rapportées par le Willem

1) Trcho Tulbberg. Ueber die Byssus des Mytilus edulis. Nova acta. Reg. Soc. Ups. Serie III. Upsala 1877.

2) Sabatier. Anatomie de la moule commune. Ann. d. Sciences naturelles. VI Serie. Tome V. pag. 1.

a) Sabatier. l. c. pag. 51 .

4) Deile Chiaje. Memorie sulla storia e anatomia degli animali etc. 1826. II. pag. 259 sqq.

5) Catrie. Ueber die Wasseraufnahme der Lamellibranchiaten. Zool. Anzeiger. 1883. Seite 560. 
Barents), soit par le picrosulfure de KLEYnenberg et l'alcool, d'après les méthodes connues (Mytilus edulis, Dreyssena, Anodonta, Unio). Où la structure histologique par la méthode de coupes n'était pas assez claire j'ai fait la macération. Toutes mes préparations ont été colorées par le picrocarmin de Ranvier et montées dans la résine de dammara.

\section{Pecten islandicus, MǘL.}

La partie antérieure du pied a une fente, qui est l'origine d'une cavité conique, dont les parois sont tapissées par un épithélium cilindrique à cils vibratiles. Immédiatement sous cet épithélium on voit des cellules avec des noyaux grands et des nucléoles bien distincts. Les corps de ces cellules sont très difficiles à distinguer les uns des autres; là pourtant où je réussis à les séparer, ils étaient ronds ou ovales, formant une masse glandulaire compacte qui est colorée par le picrocarmin.

Cette partie antérieure du pied est. séparée très-distinctement de la partie médiane. Celle-ci présente un sillon qui n'est pas en communication avec la cavité conique, mais qui est cependant tapissé par un même épithelium à cils vibratiles. A la face dorsale ou supérieure du sillon se montrent bientôt des cellules dont le protoplasme est transformé en une matière granuleuse et réfringente, qui, contrairement au noyau et à la nucléole, n'est pas colorée par le picro-carmin. Cette transformation se présente de la manière la plus évidente chez les cellules glandulaires qui se trouvent au commencement de la partie médiane. On y peut observer toutes les différentes phases. A mesure que l'on passe de la partie médiane à la partie proximale du pied les corps des cellules glandulaires sont plus difficiles à distinguer, et à plusieurs endroits d'une coupe transversale on ne voit qu'une seule masse granuleuse et réfringente. dans laquelle les noyaux sont distribués irrégulièrement. Pas à pas on peut suivre la décomposition du protoplasme des corps cellulaires et la transformation en trâinées granuleuses et réfringentes qui vont déboucher entre les cellules de la couche épithéliale.

$\mathrm{Au}$ commencement de la partie médiane on voit à la partie ventrale des coupes transversales encore des cellules conformes à celles qui bordent la cavité conique d'en haut et qui ont été nommées par CARrière »cellules muqueuses«, 》Schleimzellen«.

En s'approchant de la partie distale du pied linguiforme ces cellules disparaissent et on voit dans le sillon des filaments du byssus qui ont été coupés par le rasoir et qui tantôt ont été teints d'une couleur rougeâtre, tantôt d'une couleur jaunâtre. De plus on voit dans la partie supérieure ou dorsale du sillon se présenter des plis qui premièrement sont en plus grand nombre dans les partis latérales que dans la partie dorsale (Fig. 1). Quand les lèvres de ce sillon se sont fermées (Fig. 2), ces plis deviennent plus profonds, leur nombre se multiplie et on voit peu à peu se former (Figg. 2 et 3) une grande quantité de lamelles qui portent en saillie dans une cavité byssifère, dont la largeur surpasse bientôt la longueur, comme il est démontré par les fig. 2 et 3. Tous ces plis et les lamelles de la cavité byssifère sont tapissés par le même épithélium à cils vibratiles que nous avons trouvé dans le sillon ventral et la cavité conique. Ils sont formés par un tissu conjonctif lâche, au milieu duquel se trouvent des cellules glandulaires qui, comme les autres, vont déboucher entre les cellules de l'épithélium.

En comparant les figg. 1, 2 et 3 ou voit que des fibres musculaires sont distribuées entre la masse glandulaire. A mesure que l'on s'éloigne de la partie médiane ces muscles transversales et longitudinales en disparaissent et dans les lamelles de la cavité byssifère on ne rencontre plus de fibres musculaires.

Aboutissant à la partie postérieure de la cavité byssifère on voit les lamelles qui s'allongent et unissant la face dorsale à la face ventrale, forment de cette manière un grillage (fig. 4). L'espace entre les cloisons est occupe dans les coupes par une matière granuleuse, matière qu'on trouve quelquefois aussi dans la cavité byssifère, mais alors en plus grande quantité, durcie et. colorée quelquefois par le picrocarmin. 
C'est alors que la masse glandulaire à atteint son plus grand développement; pas à pas le nombre des cloisons et leurs dimensions commencent à s'amoindrir et bientôt on trouve les dernières traces de ces lamelles.

Enfin on ne voit plus qu'une masse glandulaire, dont les corps cellulaires se distinguent très difficilement et qui disparaît bientôt pour faire place à des tubes du foie et des organes génitaux, qui escortaient déjà depuis longtemps la masse glandulaire.

Nous avons donné à l'aide des coupes transversales une description un peu detaillée des organes byssogènes de cette espèce, parccque celle-ci, mieux qu'aucune autre, peut nous donner des idées claires et précises sur la formation d'une cavité byssifère.

L'ontogénie nous démontre une invagination ectodermique qui se glisse le long de la face ventrale du pied pour entrer dans une masse glandulaire. Cette invagination obtiendra bientôt toutes sortes d'insinuosités et de plis et s'enfoncera de plus dans la glande. La couche épithéliale qui revêtit l'invagination et ses plis fait passer entre ses cellules une substance granuleuse, la matière du byssus, qui formée en fils ou en lamelles, constitue une masse cohérente, qui, durcie dans l'eau et par l'eau, attaché l'animal à quelque corps étranger.

Tout les plis et les lamelles que l'on trouve dans la cavité byssifère sont évidemment destinés à augmenter la surface sécrétante du système glandulaire.

Etudions à présent le

\section{Pecten groenlandicus, Sowerby.}

Nous distinguons encore ici une partie antérieure ou distale, qui chez des exemplaires conservés dans l'alcool, est séparée de la partie médiane par une rainure peu accentuée à la face ventrale. Dans la partie antérieure on trouve un sillon peu profond qui se continue dans la partie médiane et est revêtu par un épithélium à cils vibratiles longs, derrière lequel se preséntent des cellules glandulaire piriformes, dont le noyau et le nucléole sont toujours situés dans la partie la plus large.

En passant de la partie distale à la partie médiane ces cellules commencent à se grouper dans la périphérie pour faire place à des cellules, dont les corps cellulaires sont très-difficiles à distinguer; elles sont très transparentes; le picro-carmin -colore très peu le protoplasme; au contraire le noyau et le nucléole à cause de leur couleur carmin-foncé sont bien visibles. Le corps cellulaire a un aspect comme si le protoplasme s'est transformé en une matière très-finement granuleuse, qui n'est plus colorée par le réactif. Ce sont ces cellules qui sécrètent la matière byssale.

Quand les lèvres du sillon se sont soudées, on entre dans la cavité byssifère et l'on voit se former de la manière la plus irrégulière des plis et des insinuosités (figg. 5 et 6 ).

Comme chez le. P. islandicus ces plis et ces lamelles sont formés d'un tissu conjonctif lâche, dans lequel sont éparses des cellules byssogènes et quelque fois on peut observer très distinctement comment ces cellules débouchent entre l'épithélium à cils vibratiles, qu'on retrouve à tous ces plis ou lamelles, par des traînées réfringentes, qui ne sont que des simples prolongements des cellules.

Comparant la cavité byssifère du P. groen la n di c u s avec celle de l'espèce précédente, cellelà est plus irrégulière et possède les indices les plus certains qu'elle est moins développée.

Passons maintenant en revue les résultats des autres naturalistes, qui ont étudié les organes byssogènes chez le genre $\mathrm{Pecten}$.

MüLler ${ }^{1}$ ) décrit chez le P. varius une fente dans la partie antérieure du pied linguiforme qui n'est pas en communication avec le sillon de la face ventrale. Ce sillon a des plis longitu-

1) MÜLLER. l. c. pag. 30 . 
dinaux qui communiquent avec une petite cavité byssifère. Puisque MüLlen s'est borné à une simple dissection il ne nous a donné aucun détail histologique.

CARrière 1) a trouvé chez Pecten sp. des Philippines et chez P. Jacobaeus dans la partie antérieure une cavité coniforme bordée par un épithélium et qui est entourée par des glandes muqueuses $\gg$ Schleimdrüsen $\ll$.

Cette cavité ne communique pas avec un sillon qui se trouve chez les espèces mentionnées sur la face ventrale et qui de même est bordé par un épithélium et entouré de glandes byssogènes. Tandis que chez une des espèces ${ }^{2}$ ) il y a une cavité byssifère dont les plis et les cloisons font saillie dans elle et sont revêtus d'une couche épithéliale à cils vibratiles, chez P. J a c o b a e u s et une autre espèce également originaire des Philippines, la cavité byssifère est reduite à des proportions plus petites.

TH. Barrors ${ }^{8}$ ) décrit chez le $\mathrm{P} . \mathrm{maximus} \mathrm{une} \mathrm{ouverture} \mathrm{à} \mathrm{l'extrémité} \mathrm{antérieure} \mathrm{et} \mathrm{libre}$ du pied, qui donne naissance à une cavité en forme de cornet, qui ne communique pas avec un sillon, qui se trouve sur la face supérieure (?) du pied. Ce sillon s'enfonce dans une cavité irrégulièrement cilindrique, qui se termine à la jonction du pied proprement dit et de la bosse de Polichinelle. L'épithélium qui tapisse cette cavité n'a pas de cils vibratiles. Immédiatement sous cet épithélium est située une couche de cellules qui se colorent en jaune foncée, par le picrocarminate d'ammoniaque, mais dont il n'a pas pu élucider d'une façon certaine la structure. Dans la partie médiane BARrois trouve des cellules piriformes qui prennent une couleur très foncée en présence du carmin de BEALE et du picrocarmin de RANvier. Les prolongéments des cellules viennent déboucher entre les cellules épithéliales du sillon.

Dans la partie proximale CARrière a trouvé une cavité plus ou moins irrégulière tapissée du même épithélium cilindrique et munie de glandes. Il n'a pu voir aucun muscle spécial qui prenne attache sur les parois de cet appareil glandulaire. Au contraire, aussi bien chez le P. groenlandicus (fig. 6) que chez le P. islandicus, un muscle spécial (m. byssale) s'insère sur l'apparẹil glandulaire comme nous l'avons aussi constaté chez la plupart des autres espèces étudiées qui suivent et qui étaient pourvues d'un byssus.

Résumant les résultats obtenus par l'étude des organes byssogènes dans le genre Pecten, il nous paraît évident, que l'appareil glandulaire du P. is landicus est le plus développé et peut être signalé comme le prototype en ce genre. Chez les autres espèces le même appareil sans doute est entré en régression d'après les conditions extérieures dans lesquelles est placé l'animal. Quelques-unes des espèces produisent un byssus bien développé comme le $P$. is landicus, le P. sanguineus (sp. des Philippines?) d'après Carrière et le P. varius selon MüLler. Chez le P. groenlandicus nous n'avons pu remarquer chez tous les exemplaires, qu'un simple fil, tandis que le P. ja cobaeus (d'après CARrière) et le P. maximus (d'après Barrois) ne possèdent pas de byssus.

Donc des 5 espèces étudiées trois ont un byssus.

\section{Lima elliptica, JEFFREYs.}

A l'extrémité antérieure on voit dans les premières coupes se former un sillon qui, au commencement, est peu profond, mais qui bientôt s'élargit et forme un canal semi-lunaire. Tout le canal est tapissé par un épithélium à cils vibratiles très-longs derrière lequel se trouvent des

1) Carrière. Die Drüsen im Fusse der Lamellibranchiaten. Arb. aus dem Zoöl. Zoöt. Instit. zu Würzburg. V. I. Heft.

2) MüLlen l. c. pag. 6 .

з) Th. Barrors. Note sur les glandes du pied de P. maxi mu s. Bulletin scientifique du Dép. du Nord. 2e série. II. page 7 . 
cellules glandulaires d'une forme ronde et ovale munies d'un noyau et d'un nucléole, qui sont parfaitement colorées par le picrocarmin. A mesure que l'on s'éloigne de l'extrémité antérieure on voit les corps cellulaires se transformer en une matière granuleuse et réfringente qui ne se colore plus par le picrocarmin. Le sillon devient de plus en plus profond et les cellules se groupent autour de lui. Bientôt il n'est plus possible de distinguer les corps cellulaires les uns des autres, puisque tous se sont transformés en une matière granuleuse jaunâtre qui constitue des traînées réfringentes et granuleuses qui viennent déboucher entre les cellules épithéliales.

Quand les lèvres du sillon se sont unies il se présente une cavité byssifère, très petite, obstruée par de la matière byssale.

Dans la paroi de cette cavité on voit les mêmes cellules glandulaires. La cavité n'a pas de plis ni de cloisons; c'est une simple cavité de forme ronde. Quand dans les coupe tranversales et longitudinales de cette cavité la lumière a disparu, les aussi cellules glandulaires disparaissent bientôt, démontrant que la masse glandulaire ne s'étend pas loin au delà de cette cavité.

CARrik̀re ${ }^{1}$ ) a trouvé chez la Lima hians une cavité byssifère avec quelques lamelles ou cloisons; il paraît donc que les organes byssogènes et particulièrement la cavité byssifère chez la Lima elliptica est moins développée que chez la L. hian s. Ni Carrière ni moi nous n'avons trouvé dans la partie antérieure des cellules morphologiquement différentes des cellules glandulaires.

\section{Modiolaria discors, $\mathrm{L}$.}

Le pied linguiforme (Spinnfinger) de cette espèce a une grande ressemblance avec celui de Mytilus edulis. Le byssus est bien grand et l'orifice de la cavité fait sortir une masse de fils soyeux qui s'attachent à un tronc de byssus bien développé.

Déjà les premières coupes transversales nous montrent des cellules à grands noyaux bien colorés. Les corps cellulaires au contraire ne sont que légèrement colorés en rose et très-finement granuleux. Avant que dans les coupes suivantes on voie surgir à la face ventrale les premières traces d'un sillon, déjà plusieurs de ces cellules ont transformé leurs corps cellulaires en une masse granuleuse et réfringente qui n'est plus colorée par le picrocarmin. Dans cette masse on voit alors distribués les noyaux, reconnaissables à leur teint rouge-carmin.

Bientôt le sillon apparaît et alors des masses granuleuses sont disséminées parmi des cellules glandulaires colorées à noyaux distinctement visibles, qui environnent le sillon et démontrent de la manière la plus décisive, que les cellules qui se trouvent dans la partie antérieure du pied sont les avant-coureurs des cellules byssogènes et de la même nature. En étudiant une seule coupe on peut observer souvent toutes les phases de dégradation par lesquelles les cellules colorées se transforment en cellules byssogènes. Chez aucune espèce je n'ai vu si positivement que des traînées formées de petites granules, débouchent entre les cellules épithéliales à cils vibratiles qui revêtent le sillon. Dans plusieurs de mes coupes (j’ai dessiné dans Fig. 7 le $n^{\circ}$. 67) on voit comment le fil de byssus qui se trouve dans le sillon, est formé par ces traînées de matière granuleuse.

Une pareille masse glandulaire côtoie le sillon jusqu’à la cavité byssifère et entre dans les parois de celle-ci. Cette cavité (Fig. 8) se présente déjà dans des coupes transversales alors que le sillon est encore peu profond, démontrant ainsi qu'elle s'allonge en avant dans le pied linguiforme. On aperçoit de plus qu'elle est divisée par une cloison médiane en deux parties congruentes (Fig. 8 et 10). Cette cloison médiane et toutes les autres sont tapissées par une couche épithéliule conforme à celle du sillon et constituées d'un tissu conjonctif très-lâche, entre les interstices duquel se trouvent des cellules glandulaires complétement identiques à celles que nous avons trouvées bordant le sillon (Fig. 9). Etudiant les coupes suivantes on voit se fermer les lèvres du sillon. 
On passe à la partie proximale du pied, donc à l'orifice de la cavité byssifère. Dès que ces lèvres se sont unies on peut distinguer entre une cavité byssifère primaire et secondaire, la dernière étant un prolongement ventral de la première. On voit alors comment cette cloison médiane qui au commencement était attachée en haut et en bas, quand la cavité secondaire s'est formée, se fait libre en bas et avec cette extrémité flotte dans cette cavité.

Peu à peu des plis se présentent dans cette cavité secondaire et la cloison s'allonge ${ }_{2}$ de sorte que bientôt sa partie inférieure est de nouveau soudée au côté ventral (Fig. 10). Un même épithélium tapisse les deux cavités.

Mais la masse glandulaire qui borde cette cavité secondaire a des cellules dont les corps cellulaires sont très transparents et très finement granuleux et paraissent être à la première vue des cellules adipeuses; le picrocarmin ne les colore que faiblement, leurs noyaux au contraire sont très-bien colorés. La masse glandulaire qui entoure la cavité primaire par une forte bande se continue le long de la masse glandulaire de la partie secondaire (Fig. 10). Longtemps j'ai douté de la différence morphologique et histologique des cellules glandulaires des deux parties. Après des recherches minutieuses sur des masses macérées et colorées je ne crois pas qu'il faille faire une différence entre les cellules de tout le système glandulaire qu'on trouve dans le pied linguiforme.

En pratiquant des coupes transversales qui de plus en plus s'éloignent de l'orifice de la cavité, il est facile de remarquer que la cavité secondaire diminue peu à peu et qu'elle est déjà disparue quand la cavité primaire avec la cloison médiane est encore passablement grande. Pas à pas enfin, toutes les cloisons ou lamelles, y compris la lamelle médiane, deviennent plus minces, la lamelle médiane ni par largeur ni par longueur ne se relève des autres et à la fin on les voit toutes diparaître comme chez le $\mathrm{P}$. is landicus dans une masse glandulaire à laquelle sont attachés les muscles byssales. Toutefois ces muscles byssales se présentent déjà dans la première coupe après que la cavité secondaire a disparu. Plusieurs fois entre les lamelles de la cavité nous avons observé des lamelles de la racine du byssus qui avaient été enlevées par le rasoir.

\section{Mytilus edulis, L.}

Les organes byssogènes de cette espèce ont été étudiés par Trcho 'Tullberg ${ }^{1}$ ) et CARRIغ̀re ${ }^{2}$ ). Ces deux naturalistes ont trouvé dans le pied linguiforme une masse glandulaire qui s'étend de la partie antérieure jusqu'à la partie proximale le long des deux bords du sillon qui se trouve à la face ventrale du pied.

Tuluberg a distingué dans cette masse glandulaire une partie qui n'est pas colorée par le picrocarmin »die grüne Drüse« et une partie qui se colore bien »die weisse Drüse«. Dans chaque moitié d'une coupe transversale l'on voit que la masse glandulaire verte à côté du sillon semilunaire se bifurque en une bande qui côtoie le vaisseau sanguin médian et une bande qui côtoie le vaisseau latéral. L'espace libre entre ces deux bandes est remplie par la glande blanchâtre. Toutefois, comme le dit l'auteur, les limites entre ces deux parties ne sont pas nettement tracées. De plus la partie blanche aussi bien que la partie verte se compose de cellules glandulaires, qui ont la forme d'une larme batavique et qui sont très-granuleuses et réfringentes. Les glandes vertes débouchent entre les cellules à cils vibratiles de la couche épithéliale, dont le sillon est tapissé, par des traînées réfringentes qui par un fort grossissement semblent être composées de granules de diverses dimensions. Pourtant on peut aussi observer dans la partie verte des tubes cilindriques, tapissés également par une couche épithéliale autour de laquelle on ne voit qu'une masse granuleuse ou les mêmes traînées décrites en haut. Ces tubes cilindriques - (fig. 11) se divisent et concourent vers le commencement $d u$ sillon, vers la fente transverse signalée par Sabatier et les autres

1) Tullberg. 1. c.

2) Carritke. Die Drüsen etc. 
auteurs comme »porus aquiferus« (fig. 13). La masse granuleuse qui se trouve dans ces tubes cilindriques est originaire des glandes qui avoisinent les tubes. Par des prolongements effilés qui se continuent en des traînées granuleuses ces cellules glandulaires débouchent entre les cellules épithéliales dans ces tubes. Quand Tullberg dit que les masses granuleuses dans les tubes »wahrscheinlich mit den Kolben in Zusammenhang stehen«, plusieurs de mes préparations m'ont donné la certitude, que la glande sécrète sa matière granuleuse aussi bien dans le sillon que dans ces tubes, qui ne sont que des invaginations du sillon.

Carrik̀re ${ }^{1}$ ) a mis en doute si les glandes verte et blanche étaient deux glandes distinctes. D'après cet auteur elles ne seraient qu'une seule glande, dont les cellules se trouveraient en différentes phases de dégradation. Je puis confirmer cette opinion. En étudiant une série de coupes consécutives on voit (fig. 11) que la masse glandulaire verte et blanche s'entrelacent et se mêlent si confusément et si irrégulièrement, que des cellules glandulaires vertes se trouvent à côté de cellules glandulaires blanches, quand, se fiant à la distribution comme on l'a observée chez la coupe précédente, on croirait ne devoir trouver que des cellules blanches.

Tullberg a donné de la cavité byssifère avec toutes ses cloisons ou lamelles une description détaillée anatomique à laquelle je n'ai rien à ajouter. Il a vu que toutes ces cloisons sont tapissées par un épithélium, à cils vibratiles derrière lequel se trouvent des cellules glandulaires qui sont insérées entre les mailles d'un tissu conjonctif très lâche. La masse glandulaire qui entre dans les lamelles et les parois de la cavité n'est qu'un prolongement de la masse glandulaire verte que nous avons déjà rencontrée et je considère aussi les glandes que Tulaberg a dessinées dans sa Fig. $1 \mathrm{k}$ et dont CARrIk̀re fait aussi mention, comme absolument identiques avec les cellules glandulaires blanches.

Résumant, nous constatons la présence d'une masse glandulaire, qui s'étend de la fente transverse jusque dans la cavité byssifère qu'elle environne. Les cellules glandulaires sont colorées par le picrocarmin quand le protoplasme n'a pas encore subi la transformation particulière, qui le rend propre à sécréter des traîneés granuleuses entre les cellules épithéliales du sillon ou des tubes cilindriques.

Cependant nous devons distinguer avec CARrıère dans la partie la plus antérieure un groupe de cellules dont le protoplasme et le noyau se colorent par le picrocarmin. Le protoplasme est très finement granuleux. La grandeur de ces cellules varie entre $13-40 \mu$, les cellules byssogène s blanches ou vertes étant toujours de 40 à $60 \mu$. CARrrère en les nommant 》Schleimdrüsenzellen« (cellules muqueuses) dit qu'elles ont un noyau qui géneralement est situé à la périphérie 》wandständig.« Un simple coup d'oeil sur la fig. 12 nous apprendra qu'il ne faut pas attacher trop d'importance à cette propriété; du reste je suis d'accord avec ce naturaliste quand il dit qu'il ne croit pas qu'ils soient en rapport avec la formation du byssus, car il y a toujours un espace entre la couche épithéliale et la masse glandulaire, qui est remplie par du tissu conjonctif. et des muscles.

Nous avons écrit plus haut que Tullberg a observé que le sillon et les tubes cilindriques aussi bien que les lamelles de la cavité byssifère sont tapissés par un épithélium à cils vibratiles. CARRIk̀re a parlé douteusement de leur présence dans les tubes et sur les parois et les lamelles de la cavité byssifère. Tullberg dans un mémoire postérieur ${ }^{2}$ ), soutenant la présence de cils vibratiles, remarque qu'il n'est pas difficile d'observer leur présence, en pratiquant des coupes minces à travers la cavité byssifère d'un animal vivant. En isolant un morceau d'une cloison, il l'examinait dans de l'eau de mer et il a observé le jeu des cils de la manière la plus positive. Puisque je n'étais pas bien sur dans mes préparations de la présence de cils vibratiles, j'ai tâché d'imiter la méthode d'obsèrvation du savant distingué, lors de mon séjour à la station zoölogique

1) Carrikire. 1. c. pag. 5.

2) Tullberg. Studien über den Bau und den Wachsthum des Hummerpanzers und der Molluskenschalen. Kongl. Svensta Vetenskaps Akademiens Handl. Bd. 19. No. 13. p. 30. Note 2. 
de la société Néerlandaise de Zoölogie. (Août 1884) à Flessingue, mais à diverses reprises je n'ai pu constater leur présence. N'osant nier leur présence je n'ose non plus certifier leur absence sur les parois et les cloisons de la cavité byssifère.

\section{Mytilus pellucidus, Pens.}

Les organes byssogènes sont identiques à ceux de M. edulis. L'extrémité antérieure du pied linguiforme a les mêmes cellules muqueuses qui, comme chez le M. e $\mathrm{dul}$ is disparaissent dès que se montre le sillon ventral. A côté de ce sillon on voit une masse glandulaire constituée de cellules de la même forme que chez le M. edulis. Cette masse se continue quand le sillon mêne dans la cavité byssifère avec ses cloisons pliées en toutes sortes de lignes recourbées.

Bien que j'aie pu constater la présence d'une couche épithéliale à cils vibratiles dans les tubes cilindriques (fig. 13) je ne les ai pu observer sur les cloisons de la cavité byssifère. Mais outre qu'une matière granuleuse débouche entre les cellules épithéliales et par les tubes dans la fente transverse, comme chez le M. edulis, j'ai observé qu'elle prend encore un autre chemin pour former des fils de byssus. Nommément on voit au sommet des deux lèvres qui bordent le sillon ventral, se former un petit canal tapissé par la même couche épithéliale qui revêtit le sillon, à travers laquelle couche une masse granuleuse et réfringente se jette dans ce canal pour former un fil de byssus très distinctement visible. Chez trois des 4 objects sur laquelle j'ai pratiqué des coupes transversales la présence de ce canal était certaine, chez le 4 ième individu sa présence était probable.

\section{Saxicava rugosa, Penn.}

Le pied linguiforme de cette espèce a un byssus bien développé qui sert à attacher l'animal au fond d'un trou qu'il s'est creusé lui-même.

Etudiant l'anatomie microscopique à l'aide de coupes transversales de l'extrémité tout à fait antérieure à la partie postérieure, nous voyons dans les premières coupes des cellules ovalaires et arrondies qui sont colorées en rose par le picrocarmin, le plasma étant très-finement granuleux. Le noyau est coloré foncé. Bientôt nous observons comment ces cellules, qui occupent alors toute la coupe, commencent à se transformer. Leur plasme devient granuleux, des granules réfringentes de grandeur variable apparaissent qui ne sont plus colorées par le réactif. Il est facile de poursuivre de phase en phase dans une seule coupe cette dégradation, par laquelle un tas de cellules deviennent une masse granuleux dans laquelle les noyaux sont dispersés. En effet les limites des corps cellulaires alors ne se peuvent plus tracer. On voit des corps cellulaires de dimensions variables éparpillés dans une masse granuleuse et réfringente dans laquelle des granules demi-rouges et demi-jaunes (fig. 20) sont les dernières traces des noyaux détruits.

Cette dégradation s'accomplit déjà dans le milieu d'une coupe transversale, alors que rien n'est encore visible qui ressemble à un sillon.

Bientôt on voit apparaître une petite invagination à la face ventrale du pied et c'est autour de cette invagination que commencent à se grouper ces cellules dégradées, décrites en haut.

A mesure que ce sillon avec son canal semi-lunaire devient profond on voit s'agrandir la masse granuleuse; cependant on $\mathrm{y}$ voit toujours éparpillées çà et là des cellules non transformées. Ordinairement la masse granuleuse est ici bordée extérieurement par une bande de cellules. non-transformées mais colorées.

L'étude des coupes suivantes nous montre un sillon qui s'enfonce rapidement dans la masse glandulaire et bientôt la largeur du sillon est telle qu'on s'approche de l'entrée de la cavité byssifère. Alors du fond du sillon on voit surgir deux plis (fig. 15) qui bientôt s'élevant de plus en plus (fig. 16) divisent l'orifice de la cavité byssifère en deux moitiés. Après que ces deux 
plis se sont soudés et que les lèvres du sillon se sont unies (fig. 17), on voit la cavité byssifère divisée en deux culs-de-sac, qui sont complètement isolés l'un de l'autre (fig. 18).

En poursuivant par des coupes traversales l'étendue de cette cavité byssifère double, on voit que la cloison ou la lamelle médiane de la cavité s'épaissit de plus en plus, tandis que la lumière de la cavité s'amoindrit. Mais le nombre des plis ou des lamelles commence à s'agrandir et les muscles postérieures du byssus, qui depuis longtemps étaient visibles dans les coupes transversales, (fig. 15-19) deviennent de plus en plus volumineuses et s'insèrent de tous côtés dans la masse glandulaire.

Th. Barrors ${ }^{1}$ ) a étudié la même espèce et a donné une exposition sommaire de ses résultats qui quant à la grosse anatomie concordent avec les miens. Cet auteur pourtant distingue deux systèmes de glandes, l'une se colore très difficilement sous l'influence des réactifs, est d'un noir verdâtre et entoure le sillon de toutes parts dans une coupe faite à l'extrémité tout à fait antérieure $\mathrm{du}$ sillon. Un millimètre plus loin Barrors voit des glandes blanches tandis que les glandes blanches sont considérablement restreintes. Les cellules des glandes noires sont très granuleuses et fortement pigmentées; malgré les recherches les plus minutieuses, Barrois n'a $\mathrm{pu}$ trouver leurs concluits excréteurs. Contrairement les cellules de la glande blanche ont leurs corps moins granuleux et plus transparents et se colorent bien sous l'influence des réactifs. Sous forme de traînées réfringentes leur conduits excréteurs viennent s'ouvrir entre les cellules épithéliales $d u$ sillon et du canal similunaire. Outre ces deux systèmes glandulaires Barrors distingue encore des glandes blanches, qui sont disséminées dans le tissu conjonctif qui environne la cavité byssifère. Ces cellules glandulaires sont fort granuleuses, réfringentes et ne se colorent pas aussi bien que les glandes $d u$ sillon; elles se trouvent à la hauteur du canal du byssus en avant de l'endroit où il se bifurque et donne naissance aux deux culs-de-sac.

Une étude minutieuse des cellules glandulaires dans les différentes régions du pied, aussi bien à l'aide des coupes que par macération, m’ont donné la conviction quil n'y a pas lieu de distinguer trois systèmes glandulaires distincts. Déjà Barrors cependant a douté que les glandes blanches et la glande noire dans les parties médiane et distale du pied constituent deux systèmes différents. 》Ils ne diffèrent 》dit-il« en un mot que par la pigmentation. Si l'auteur avait étudié aussi des espèces draguées dans d'autres mers, il n'aurait pas, j'en suis certain, hésité à résoudre la question. D'après. mes recherches il n'y a qu'un seul système de glandes cellulaires qui va d'une extrémité du pied à l'autre, entourant la cavité byssifère et se montrant dans ses cloisons ou lamelles. Par des traînées granuleuses et réfringentes le système glandulaire débouche dans le sillon et dans la cavité, toujours entre les cellules épithéliales à cils vibratiles dont sont tapissés aussi bien le sillon que la cavité byssifère.

\section{Arca septentrionalis, Sars.}

En étudiant les organes byssogènes d'abord par dissection anatomique, on voit sortir à la fin d'un sillon ventral qui mène à une cavité byssifère un seul fil de byssus. Si nous poursuivons ce fil il se bifurque et nous apercevons une cavité byssifère qui par une masse musculaire cunéiforme est divisée en deux parties, qui sécrètent chacune un fil de byssus dont les extrémités antérieures s'unissent en forme de $\mathrm{V}$ pour former le seul fil que nous avons observé (fig. $21 \& 22$ ).

Si nous pratiquons des coupes transverses d'une extrémité du pied à l'autre, nous voyons dans les prémières coupes déjà se montrer des cellules rondes ou polyédrales qui se colorent peu par le picro-carmin, excepté le noyau. Ces cellules sont disséminées dans les interstices d'un tissu

1) Th Barkors. Notes sur les glandes du byssus de Saxicava rugosa. Extr. du Bull. scientif. d. J)ép. du Nord II Serie. II année, pag. 9 et 10. 
conjonctif très-lâche. Bientôt le nombre de ces cellules s'agrandit, le tịssu conjonctif disparaît et nous rencontrons une masse glandulaire, dont les corps cellulaires ne sont pas facilement à distinguer. Successivement on voit s'accomplir des transformations dans les cellules qui sont situées dorsalement du sillon. De petites granules réfringentes, qui ne sont plus colorées par le réactif déforment le protoplasme de quelques cellules, dont le nombre s'agrandit peu à peu et bientôt ces cellules à protoplasme granuleux se groupent à droite et à gauche du sillon.

Le sillon devenant plus profond, l'étude des coupes suivantes (fig. 23, 24, 25) nous démontre comment le sillon mène dans une cavité byssifère et que du fond d'elle s'élève une masse musculaire cunéiforme. Par cette masse la lumière de la cavité est divisée en deux fentes larges (fig. $24 \alpha$ et $\beta$ ). Un épithélium à cils vibratiles tapisse le sillon et la cavité décrits. Derrière l'épithélium des deux fentes ( $\alpha$ et $\beta$ ) mais toujours extérieurement on voit une masse glandulaire s'étendre le long du sillon. A mesure que dans les coupes la hauteur de la masse cunéiforme aceroît, l'étendue de la masse glandulaire décroît, comme il est demontré en comparant les fig. 24 et 25. Cependant les lèvres du sillon s'unissent et les dernières traces de la cavité disparaissent et avec elles les cellules glandulaires éparses. Comme chez les autres espèces étudiées les cellules glandulaires se trouvent immédiatement derrière l'épithélium, débouchant par des prolongements effilés, qui se changent en traînées granuleuses entre les cellules de la couche épithéliale dans le sillon et la cavité byssifère (fig. 26).

La disposition générale des organes byssngènes chez cette espèce concorde assez bien avec celle de A. barbata. MüLler ${ }^{1}$ ) dit de cette espèce $\gg$ Der zungenförmige Muskel ist etwas breit und dick. Er ist der Länge nach zusammengefaltet. wie der Fuss der Gastropoden, enthält auch ịn der Mitte eine der Länge nachgehende Vertiefung, aber die scharftiefe Längsfurche fehlt ihm. Die Byssushöhle hat einen sehr weiten Zugang. Auf ihrem Boden ist in der Mitte eine fleischige Erhöhung, von welcher ringsum Furchen nach der elliptischen Peripherie ablaufen«.

D'après CARRIÈre ${ }^{2}$ ) A r ca $\mathrm{N}$ o a e a une cavité byssifère avec plusieurs plis lamellaires. Le sillon et la cavité avec ses plis sont environnés de cellules glandulaires entre lesquelles ce naturaliste distingue des cellules rondes qui débouchent dans le sillon et des cellules pyriformes qui sécrètent une matière granuleuse dans la cavité byssifère. Chez le A r c a gran os a ce naturaliste a observé un sillon qui cependant n'est pas entouré de cellules glandulaires, seulement dans la partie la plus postérieure, il a trouvé les traces de lamelles byssogènes et quelques cellules glandulaires à l'orifice de la cavité byssifère rudimentaire.

TH. Barrois ${ }^{3}$ ) a étudié les organes byssogènes de A. tetragona. Ce naturaliste a trouvé un sillon nettement délimité en deux parties qui ont chacune leur structure spéciale. Il a observé des glandes brunes qui viennent déboucher dans la partie antérieure et un peu dans la partie postérieure et de plus des lamelles au nombre de 20 environ, tapissées par un épithélium et constituées de tissu conjonctif au milieu duquel sont disséminées des glandes en grappes qui, comme les autres, vont déboucher entre les cellules épithéliales. Ces lamelles se trouvent dans une cavité byssifère de très peu d'étendue, dans laquelle ces lamelles sont formées d'une partie fondamentale de tissu conjonctif, d'une partie musculaire et d'une partie glandulaire. Les cellules glandulaires de cette dernière partie sont plus petites que celles des glandes brunes, granuleuses et réfringentes; elle se colorent facilement par le réactifs. Je ne discuterai pas ici l'opinion de l'auteur distingué que ces deux ordres de glandes auraient des fonctions tout à fait spéciales. Je me propose d'aborder cette question dans les »Conclusions générales«. Seulement je veux fixer l'attention sur la dégradation des organes byssogènes, comme elle est visible dans les quatre espèces du même genre.

1) MüLLLR. l. c. pag. 24.

2) Carrière. Die Drüsen etc. pag. 8 et pag. 15 .

3) Th. BarroIs. Extr. etc. II série. II année. $\mathrm{N}^{\circ} .8$. pag. 278. 
Chez l'A r ca tetrag on a il y a une cavité byssogène avec plusieurs lamelles qui sécrètent une matière byssogène. Comme l'a déjà prononcé BARrors, il n'est pas douteux que les lamelles musculaires de l'A r ca $\mathrm{N}$ oa e sécrètent aussi une matière qui sert à former le byssus, bien que CARRIÈre n'ait pas pu constater la présence de glandes dans ces lamelles.

Chez Arca septentrionalis on ne trouve qu'une simple lamelle musculaire, notre masse musculaire cunéiforme (fig. 24 et 25) - qui pend dans la cavité byssifère mais qui ne possède pas de glandes. L'Arca granosa enfin a bien un sillon à la face ventrale du pied mais sans que des cellules glandulaires bordent ce sillon. Quelques lamelles se présentent et la cavité byssifère, quoique très peu développée, nous montre quelques restes de glandes situées là où le sillon touche à cette cavité.

\section{Dreyssena polymorpha, van Ben.}

MüLler ${ }^{1}$ ) a donné une description du pied linguiforme et de la cavité byssifère sans entrer toutefois dans des détails histologiques.

CARRIÈre a étudié cette espèce au point de vue de la structure histologique des glandes qui bordent le sillon et la cavité dans laquelle le sillon se termine.

Pour fixer les idées, j'ai dessiné le pied linguiforme avec la bosse de Polichinelle (fig. 27), les lignes $a b, c d$ et ef correspondant avec les coupes fig. 28, fig. 29 et fig. 30.

Avec Carrière j'ai trouvé dans l'extrémité tout a fait antérieure, des cellules glandulaires qui diffèrent des cellules byssogènes sensu strictiori. Les premières coupes sont tout à fait remplies par ces cellules. Légèrement colorées par le picrocarmin elles ont un noyau et un nucléole, bien distincts et sont d'une forme ronde ou ovalaire. Dès que le sillon se présente les premières cellules byssogènes se montrent aussi. Un simple coup d'oeil sur la fig. 31 nous fait apercevoir que les dimensions des cellules byssogènes et des cellules muqueuses (Schleimzellen de CarrIÈrE) diffèrent, que les cellules byssogènes sont plus grandes, très granuleuses à grands noyaux et à nucléoles. Aussi les unes comme les autres sont disseminées dans un tissu conjonctif très lâche.

La glande byssogène en s'agrandissant et en entourant le sillon, qui devient de plus en plus profond, pousse les cellules muqueuses vers la périphérie, qui bientôt disparaissent. Tandis que le sillon entre plus profondément dans la glande, les lèvres du sillon s'épanouissent (fig. 29) mais les dimensions de la glande décroissent. On trouve ici dans les coupes les première traces des muscles de byssus. C'est alors qu'on voit se dessiner au fond du sillon des plis longitudinaux (fig. 29) qui, au commencement, ont peu de hauteur. Peu à peu ces plis commencent à se marquer plus profondément et après quelques coupes, surtout quand les lèvres se sont soudées, on voit au côté dorsal de la cavité une douzaine de plis ou de lamelles, qui, comme toute la cavité, sont tapissés d'une couche épithéliale.

Ces lamelles sont formées par du tissu conjonctif et des cellules glandulaires de la même espèce que celle que nous avons trouvée comme bordant le sillon. Pratiquant des coupes à travers la base de la cavité, je vois encore toujours des cellules glandulaires dans les lamelles; à ce sujet donc je suis en désaccord avec CARRIère qui dit, que la glande byssogène ne s'étend pas jusqu’à la fin de la cavité. Enfin la lumière de la cavité commence à s'amoindrir. Les muscles du. byssus s'insèrent de plus en plus sur la base des lamelles. Encore quelques coupes et on ne voit plus que des masses musculaires interceptées par quelques fentes très minces tapissées par la couche épithéliale, les dernières restes de la cavité byssifère avec ses lamelles de différente grandeur.

1) Müller. 1. c. pag. 15.

2) Carrière. Die Drüsen ete. pag. 7. 


\section{Cardium edule, L.}

Th. Barrors $\left.{ }^{1}\right)$ a etudié cette espèce par des coupes transverses et longitudinales aussi bien que par dissection. Il a observé un sillon qui s'étendait sur presque tout le bord inférieur du pied, depuis la pointe jusqu'à quelque distance du talon. Il a constaté un sac d'un blanc opaque et éclatant et qui est maintenu dans la cavité pédieuse par des ligaments de tissu conjonctif et donne insertion à quelques muscles. De sa partie antérieure et supérieure se détache un canal qui se rend à l'extrémité du sillon, la plus rapprochée du talon. De ce canal s'echappe le byssus qui est secreté par la grosse glande et deux glandes accessoires ou prolongements glandulaires, de même nature que la glande elle même.

Ces deux glandes accessoires viennent, après avoir suivi le trajet du canal du byssus, s'appliquer à droite et à gauche de chaque côté de la fente.

Il me semble que l'auteur distingué n'a pratiqué des coupes que pour contrôler les résultats qu'il avait obtenus par dissection. J'ai suivi un chemin tout contraire. J'ai commencé par faire des coupes transverses et longitudinales, et plus tard j'ai contrôlé par dissection l'exposé de la structure anatomique et histologique de l'intérieur du pied que je m'avais fait.

De cette manière j'ai trouvé dans les coupes les plus antérieures du pied (fig. 32), entre les mailles d'un tissu conjonctif très lâche ${ }^{2}$ ), des cellules d'une forme ronde ou ovalaire avec un noyau et un nucléole très-distincts. Le corps cellulaire était finement granuleux et n'était 'coloré que très-faiblement. Très peu en nombre dans les premières coupes, bientôt leur nombre accroit. Elles se pressent de plus en plus entre les mailles du tissu conjonctif de sorte qu'il devient très-difficile de distinguer alors leur forme particulière. A une distance d'environ $1 \mathrm{mM}$. les cellules commencent à se disperser dans la périphérie et au milieu d'une coupe transversale on voit cà et là des lacunes formées par le tissu conjonctif.

Encore un millimètre et nous observons les premières traces d'un sillon. Alors les cellules glandulaires se groupent autour du sillon et la tiennent compagnie d'un bout à l'autre. Ce sillon, qui nous montre des plis longitudinaux, n'a au commencement que peu de profondeur; dans le $\frac{3}{4}$ de sa longueur il devient subitement plus profond, et la masse glandulaire accroît en proportion. Et quand les lêvres du sillon se sont unies il ne reste qu'un canal étroit (le canal du byssus de CARrière) qui est entouré de cellules glandulaires conformes à celles que nous observions comme environnant le sillon. La lumière du canal devient peu à peu plus petite; une coupe transverse du canal a la forme d'une ellipse très allongée. On voit se montrer quelques plis et insinuosités et bientôt le canal s'est recourbé en forme d'un C aplati (fig. 33). Le nombre des plis va augmentant et on pourrait dire qu'on a ici une cavité byssifère très rudi. mentaire entourée d'une masse glandulaire en proportion très développée.

Les coupes pratiquées à cette hauteur dans le pied nous démontrent que cette cavité byssifère est avoisineé par le canal alimentaire, car chacune des coupes à cette hauteur nous laisse voir une ou plusieurs diverticules de ce canal. Peu à peu notre cavité byssifère rudimentaire devient de plus en plus étrọite pour disparaitre bientôt, mais toujours elle est bordée par un groupe de cellules glandulaires, identiques à celle dont nous avons fait mention plus haut.

Toutes ces cellules glandulaires sont un peu transparentes et réfringentes, le picrocarmin ne les colore pas ou très difficilement; ordinairement le noyau et son nucléole ne se distinguent plus. Je ne crois pas qu'il soit nécessaire de comparer en détail les résultats de Barrois avec les miens. Pour moi il est évident que les glandes moniliformes, et accessoires de BarinoIs, qui

1) Th, Barrois. Extr. d. Bull. sc. du Dép. d. Nord. II. Année. II. Série. No. 1, pag. 1.

2) Je me propose de donner plus tard ailleurs une étude sur le tissu conjonctif des Lamellibranches et les cellules de Langer. Voyez. Zeitschrift für wiss. Zoologie. XXXIX. pag. 137. 
suivant ce naturaliste se trouvent à gauche et à droite du sillon et qui deviennent plus distinctes l'une de l'autre, à mesure que la fente devient plus profonde, font avec le sac d'un blanc opaque et éclatant du même naturaliste, un seul système glandulaire. Ce sac lui-même n'est qu'un amas de cellules glandulaires perforé par le canal qui est un prolongement du sillon. Les cellules glandulaires d'après BARroIs se colorent fortement par le picrocarmin. Je ne sais pas de quelle méthode de coloration ce naturaliste a fait usage. Je veux bien exposer la mienne. Je coupais le pied de l'animal vivant, et je le traitais avec le picrosulfure de KueYn cinberg, durant cinq heures, et puis par l'alcool de $70^{\circ}$, en renouvelant jusqu'a ce que l'alcool resta blanc. Alors successivement je changeais l'alcool de $70^{\circ}$ en alcool de $60^{\circ}$. jusqu'à $40^{\circ}$. Puis le pied coupé fut mis pendant quinze jours dans le picrocarmin de Ranvien. Après cette coloration j’employai de l'alcool de $70^{\circ}$ pendant quelques jours constamment. renouvelé, puis l'alcool absolu. De cette manière tous les tissus, y compris l'épithélium à cils vibratiles qui tapisse le sillon et le canal d'un bout à l'autre, étaient colorés de la manière la plus belle, excepté toujours la masse glandulaire qui borde le sillon et le canal. Il m'a été impossible de constater la moindre différence morphologique entre les cellules glandulaires de la moité antérieure et de la moitié postérieure.

D'après Barrois les bords du sillon et son intérieur seraient couverts par des papilles pigmenteés d'un beau jaune orange. Toutefois il est connu de chaque naturaliste qui s'est occupé plus que superficiellement de l'anatomie et de l'histologie des Lamellibranchiates et des Gastropodes, que la présence d'un pigmént dans les cellules épithéliales est un pur hasard. Par exemple je possède une série de coupes de Mytilus edulis (A) dont presque toutes les cellules épithéliales sont pigmentées d'un brun froncé. D'ou il résulte que l'opinion de Barrors que ces papilles sécréteraient un mucus 'clair et filant est très peu fondée.

Dans toutes mes coupes je n'ai jamais rencontré que la lumière était à demi obstruée par un morceau des byssus enlevé par le rasoir comme nous le décrit Barrois. Peut-être il faut en chercher la cause que j'ai recueilli mes objets dans le mois d'août, un époque dans laquelle Barrors trouva moins d'individus qui étaient encore pourvus de leur byssus.

Maintefois pourtant j'ai trouvé dans le canal une matière moitié granuleuse et moitié muqueuse qui vraisemblablement était sécrétée par les cellules glandulaires à travers la couche épithéliale du sillon et du canal byssifère.

\section{Cardium ciliatum, FABR:}

Chez cette espèce on trouve à quelque distance du talon du pied un orifice qui est l'entrée d'un canal qui monte dans le tissu musculaire et conjonctif du pied. En l'étudiant à l'aide des coupes je n'ai pu constater la présence d'un sillon à sa face ventrale. Le canal en question, tapissé partout par une couche épithéliale à cils vibratiles, est bọrdé par des cellules glandulaires qui ne se colorent pas non plus par le picrocarmin et qui ont la plus grande ressemblance avec celles de C. edule. La lumière du canal qui a toujours la forme d'une ellipse allongée devient plus petit à mesure que le canal s'enfonce dans le pied, pour disparaître après quelque temps. Chez des individus adultes sa longueur n'est que de quelques millimètres.

Comparant les résultats obtenus par Carrìne chez $\mathrm{C}$. oblong u m avec ceux de Barrors et avec les miens, il me paraît que le $\mathrm{Card}$. oblongum et e dule ont leurs organes byssogènes également développés. Ces deux espèces ont un sillon ventral bordé par des cellules glandulaires, qui se continue dans un canal, également bordé de ces cellules montant dans les tissus musculaire et conjonctif du pied. Chez le C. ciliatum on ne trouve qu'un canal, qui, bordé par des cellules semblables mais en moindre quantité, nous apprend de nouveau que dans un même genre les organes byssogènes peuvent être en diverses phases de régression. 


\section{Yoldia limatula, SAY et Yoldia sapotilla, Goold. Leda pernula, Mürt. et Nucula tenuis, Mont.}

Chez aucune de ces espèces appartenant à la famille des A r ci ida è je n'ai trouvé des glandes byssogènes, bien que toutes ont un pied qui est pourvu d'une fente quelquefois très profonde et très large.

\section{Mya arenaria, L.}

Des coupes transverses pratiquées d'une extrémité du pied à l'autre démontrent que chez cette espèce qui habite nos rivages sableux et vaseux, on ne trouve ni un sillon ni un canal quelconque conduisant à quelque glande rudimentaire.

D'une autre espèce, M. byssifera, il est connu que quelquefois elle file un fil de byssus.

\section{Anodonta ventricosa, Pfeifrer et Unio batavus, Lam.}

Comme j'ai publié dans une notice préliminaire ${ }^{1}$ ) ni chez A. ventricosa ni chez U. batavu's je n'ai trouvé des cellules glandulaires, ni l'une ou l'autre cavité ou sac tapissé par une couche épithéliale à cils vibratiles.

Carrière 2) a été plus heureux, du moins sur 3 ou 4 individus dont il a fait des séries de coupes il a trouvé chez un exemplaire un tel sac à l'extrémité postérieure de la carène du pied. De 6 individus de différente grandeur je n'ai pu constater une seule fois la présence d'un tel sac ${ }^{3}$ ).

\section{Conclusions générales.}

Les recherches précédentes sur les organes byssogènes des Lamellibranchiates ont démontré, je l'espère, avec celles de CARrIk̀re et de Barrors que chez toutes les espèces étudiées par ces auteurs et par moi qui possédaient un appareil à byssus plus ou moins développé, on trouve à la face ventrale du pied un sillon qui va d'une extrémité à l'autre et qui mène dans une cavité qui à été nommée 》byssifère«.

Le sillon ventral et la cavité byssifère sont tapissés par un épithélium qui chez la plupart des espèces a des cils vibratiles. Autour du sillon et de la cavité sont groupées des cellules glandulaires dont le corps cellulaire et le noyau se sont transformé en une masse granuleuse et réfringente. Pour augmenter la surface sécrétante chez les espèces qui possèdent un byssus très développé, la cavité byssifère et quelquefois aussi le sillon ventral possèdent des plis longitudinaux qui se maintiennent dans l'orifice de la cavité pour s'agrandir peu à peu, de cette façon se developpant en des lamelles ou des cloisons qui font saillie dans la. cavité. Chez les Mytilidae et les Pectinidae le nombre de ces lamelles est considérable; chez les représentants des autres familles nous les avons observé dans une régression progressive, chez quelques unes (Car didae) le sillon est en continuation avec un canal qui perce une masse glandulaire pour se terminer en cul de sac.

1) Catrixe. Ueber die Wasseraufnahme etc. Zool. Anzeiger 1883 . pag. 560.

2) Carrtère. Die Drüsen im Fusse ete. pag. 22.

3) Que je n'ai pu constater la présence de cellules glandulaires n'a rien d'extraordinaire" dit M. BARRors. (Pori aquiferi. pag. 13. Note 2). Je le crois rolontiers. Aussi ce n'était pas pour constater quelque chose d'extraordinaire que dans ma notice préliminaire j'ai fait mention de mes résultats négatifs par regard à la présence de glandes byssogènes chez ces Unionidae. 
Les cellules glandulaires qui bordent le sillon et la cavité ont pour le plupart une forme arrondie et ovalaire ou avec un prolongement effilé, et forment des traînées réfringentes et granuleuses qui débouchent entre les cellules épithéliales, aussi bien dans le sillon que dans le cavité byssifère.

Par cette opinion qui est basée sur des observations toutes positives (fig. 9) je suis en contradiction toute péremptoire avec CARr.ère qui dit ${ }^{1}$ ) 》dass die Byssuslamellen entstehen, indem die »von den einzelnen, meist cylinderförmigen Epithelzellen abgesonderten Secretfäden mit einander 》verschmelzen« et ailleurs ${ }^{2}$ ) 》meiner Überzeugung nach werden die Byssuslamellen in den Byssus»fächern abgesondert und zwar von den Epithelzellen derselben«.

Lisant et relisant, j'ai pensé d'abord à un lapsus calami, pourtant j'ai du changer d'opinion, vu que l'auteur dit positivement $\gg$ ich stehe hier hauptsächlich mit Tuluberg im Widerspruch. Aber »seine Angabe über die Art. und Weise wie das Secret in die Fächer gelangen und sich dann

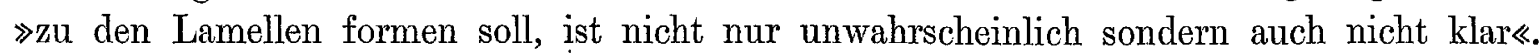

Or quelle est l'opinion de Tullberg. Nous citons ${ }^{3}$ ). $\gg$ Auch die verticalen Lamellen, die die $\gg$ Wurzel ausmachen, werden gewiss auf dieselbe Weise aus den Drüsen der verticalen Scheide»wände gebildet als der Faden aus den Drüsen der Rinne gebildet wird, d. h. zwischen den »Epithelzellen münden die Drüsen aus, durch schmale Streifen, die bei starker Vergrösserung 》aus einer Reihe stark lichtbrechenden Körnern zu bestehen scheinen.«

Un peu plus loin Tuluberg dit: »die im Stamme (des Byssus) gefalteten Lamellen, die eine Fortsetzung der Wưzellamellen sind, können nicht anwachsen, da keine absondernden Drüsenwände sich zwischen ihnen finden.

Aussi Barrors en étudiant Arca tetragona ${ }^{4}$ ) a trouvé dans les lamelles de la cavité byssifère des cellules glandulaires qui sécrètent une matière granuleuse et réfringente entre les cellules de l'épithélium.

La présence de cellules glandulaires dans les lamelles de la cavité byssifère, identiques à celles qui se trouvent groupées autour du sillon et en continuation directe avec les dernières, comme est constaté par Tullberg, Barrois et par moi, prouve que la racine, le tronc et les divers fils du byssus sont d'une même origine. Ensuite de quoi je n’accepte pas les résultats des recherches de MüLler, comme ils sont uniquement basés sur l'étude du byssus et non des organes byssogènes, c'est à dire: une distinction entre une matière filamenteuse et une matière agglutinative. Ni Tullberg, ni Carrivere n'ont suivi Müllier sur ce terrain, Barrois est le seul qui accepte cette classification insolite, chez Arca tetragona: 》Les glandes brunes qui »viennent: déboucher dans la partie antérieure du sillon et un peu dans la partie postérieure »sécréteraient la matière agglutinative; au contraire les glandes blanches; situées dans l'intérieur des 》lamelles de la cavité byssifère deverseraient leur produit de sécrétion exclusivement dans la »partie postérieure du sillon et formeraient la partie filamenteuse »die Byssusmaterie.« Me basant aussi bien sur la structure histologique des cellules glandulaires trouvées dans le pied, que sur les observations directes sur la structure. des fils de byssus qui seront décrites dans le chapitre suivant, je n'hésite pas à prononcer comme mon opinion qu'il n'y a pas lieu de distinguer deux systèmes glandulaires dans le pied des Lamellibranches, dont l'une sécréterait la matière filamenteuse et l'autre la matière agglutinative.

1) CARrière. Die Drüsen etc. pag. 4.

2) Carrière. 1. c. pag. 12.

3) Tullberg. Ueber die Byssus des Mytilus edulis 1. c.

4) Barrois. Extr. etc. II Serie. II Année. 8. pag. 278.

5) Barrors: Extr. etc. II Série. II Année. No. 8, pag. 282 et 283. 


\section{B. De la manière dont les Lamellibranches s'attachent à des corps étrangers.}

L'étude des organes byssogènes chez les espèces sus-mentionnées me fit concevoir l'idée d'observer minutieusement chez une ou plusieurs de ces espèces de quelle manière elles s'attachent les unes aux autres ou aux corps étrangers environnants.

Consultant la bibliographie il paraît que notre compatriote A. DE HEIDE ${ }^{1}$ ) a été le premier qui par observation directe s'est occupé de la manière dont la moule commune s'attache à quelque objet voisin.

Indépendamment de lui, RÉAumuR ${ }^{2}$ ) a fait à peu près les mêmes observations et une foule d'expériences avec des moules qu'il avait dépouillées de leurs fils en les coupant le plus près possible $\mathrm{du}$ bord de la coquille. Quelquefois après avoir entre-ouvert la coquille autant qu'il le put sans endommager les muscles, il introduisait dans la coquille des ciseaux avec lesquels il coupait tout le paquet ou la houppe des fils. De cette manière il a observé que le fil est formé dans la fente ventrale du pied linguiforme et qu'il s'attache à une espèce de tendon rond ou plutôt un fil de même espèce mais plus grand, que nous avons nommé tronc de byssus. 》Ce »tendon rond 'est logé dans un tuyau creux, qui forme la base de la filière et qui est proba»blement le reservoir dans lequel s'assemble la liqueur, qui forme en suite des fils, car il est »entouré de diverses parties glanduleuses propres à filtrer la liqueư gluante destinée à les com$\gg$ poser«. Si la moule veut s'attacher »elle commence apparemment par comprimer les parties 》glanduleuses qui contiennent le suc gluant sus-nommé. Ce suc comprimé des parties qui le »contenaient se rend dans le reservoir qui est à la base de la filière. Là une partie s'attache »comme à' son tronc au gros tendon qui est logé dans la même cavité. La moule ensuite fait »monter le reste de se suc dans le canal qui occupe presque toute la longueur de la filière. Le »canal étant alors formé le suc ne saurait s'en épancher, c'est sans doute pour l'y conduire qu'al»ternativement elle allonge et qu'elle raceourcit la filière un grand nombre de fois. La liqueur »étant conduite jusqu'au bout du canal, elle forme un fil visqueux auquel il ne manque plus que $\gg$ de prendre de la consistance et que d'être attaché sur quelque corps pour devenir un des fils »dont nous avons parlé. La moule alors applique sur le corps qu'elle a choisi le bout de sa $\gg$ filière, elle l'y laisse quelque temps en repos et c'est pendant ce temps que le fil visqueux acquiert $\gg$ de la consistance et qu'il se colle par son extrémité. Il est comme posé perpendiculairement $\gg$ sur le corps auquel il est adhérent et comme ce fil est un petit cylindre, la petite base de ce »cylindre est posée sur le corps auquel il est attaché. Afin qu'elle y tienne plus fortement, »la moule donne à cette base trois ou quatre fois plus de diamêtre que n'en a la reste du fil. 》Voici donc un fil moulé dans la filière, attaché par un de ses bouts au tendon qui sert de tige »commune et par l'autre bout à un corps stable. Ils ne reste plus à la moule qu'à le dégager »de la filière. Les fibres circulaires (du canal) lui en donnent la facilité, elles servent à ouvrir $\gg$ le canal dans toute sa longueur et ce canal ouvert la moule n'a plus qu'à éloigner la filière $\gg d u$ fil qui y est contenu. C'est ce qu'on lui voit faire avec vitesse, elle porte sa filière en 》arrière, presque parallèlement au nouveau fil, après quoi elle le fait rentrer dans sa coquille.«

De plus Réaumur a observé que les nouveaux fils que les moules ont formés ont toujours été collés près de l'origine de ce gros fil ou tendon rond, décrit plus haut. »Or si tous les fils que »la moule forme sont collés près de l'origine il suit de là évidemment que ce fil crôit comme $\gg$ un cheveu.«

Nous nous sommes servis autant que possible des propres paroles de RÉaumur, parcequ'elles nous donnent une idée assez précise de la manière dont la moule s'attache. Pourtant, puisque

1) A. DE HEIDE. 1. C. $\$ 29$. pag. 54 .

.) Reaumur 1. c. pag. 117. 
RÉAUMur a négligé, comme il paraît, la dissection anatomique et ne pouvait étudier la structure histologique du sillon et de la cavité byssifère, son exposé n'est pas correct dans toutes détails et notamment dans la manière dont est formé la plaque terminale par laquelle l'animal s'attache à un objet quelconque. Encore la façon dont il croit que les fils du byssus sont attachés au trone de byssus est en contradiction avec mes observations.

Grâce à un séjour prolongé en 1883 et 1884 aux bords de l'Escaut, j’ai été à même de me procurer en assez grande quantité des moules de différentes dimensions. De plus, j’ai fait les mêmes observations sur le Dreyssena polymorpha, qui s'attachent ordinairement sur des Unio et des Anodonta de nos rivières. Les résultats étant chez ces deux espèces à peu près identiques je me bornerai à décrire celles faites sur la moule commune.

Pour observer le plus commodément j'ai mis séparément dans des cuvettes de verre pleines d'eau de mer des moules de différent âge, ayant soin que l'eau restât aussi fraîche que possible. Ordinairement au moyen de petits cailloux la position de l'animal avec ses coquilles closes était telle, qu'il reposait avec son ligament sur le fond de la cuvette. Après quelque temps d'attente je vis s'ouvrir lentement les coquilles et la pointe du pied linguiforme apparut. Alors le pied commençait à tâter le voisinage, mais puisque l'animal était complétement isolé, il arrivait quelque fois que le pied s'allongeait jusquà deux ou trois fois la longueur de l'animal sans trouver quelque objet dans le voisinage. Enfin, ne trouvant comme ordinairement auprès de lui quelques autres moules, après diverses tentatives vaines, la pointe du pied se glissait le long de la coquille qui était inclinée vers le fond de la cuvette et de cette manière atteignait le fond. Durant ces tentatives la fente du pied était toujours tournée vers l'observateur ou, pour m'exprimer d'une manière plus intelligible, le pied touchait avec son côté dorsal l'épiderme de la coquille. Une fois que le pied avait atteint le fond de la cuvette, par contraction des muscles, l'animal faisait reposer la coquille, qui était inclinée vers l'horizon sur' le fond de la cuvette malgré les petits cailloux qui étaient autant d'obstacles.

Dans la plupart des cas l'animal alors en allongeant et en raccourcissant le pied sans toutefois abandonner le fond sur lequel il s'était appliqué, traînait son corps après lui. Enfin après quelque temps, le pied appliqué soit sur le fond soit sur le manteau de la cuvette cylindrique restait tranquille. Alors à l'aide d'un microscope simple on pouvait aisément observer, si le pied était appliqué sur le manteau de la cuvette, que de la fente transverse qui termine le sillon ventral s'écoulait une matière blanchâtre, qui peu à peu devenait de plus en plus opaque. On voyait se former la plaque terminale. Au commencement. la fente transversale. s'appliquait tout simplement sur le manteau du cylindre, bientôt cependant, cette fente transversale s'allongeait en forme d'un triangle équilatéral et plus abondant qu'auparavant, on voyait sortir la matière blanchâtre. Or d'après les études anatomiques et histologiques sur le système glandulaire de M. e dulis il est evident que les tubes cylindriques qui se trouvent disséminées dans la substance glandulaire $\mathrm{du}$ pied et qui débouchent dans la fente transversale du sillon ventral, versent alors leur contenu, une matière granuleuse et réfringente que nous avons rencontrée dans les tubes des coupes transversales.

Quand la plaque terminale était formée l'animal retirait le pied, un fil mince sortait du sillon qui était attaché par un de ses bouts à la plaque terminale et par l'autre au tronc de byssus.

Le temps nécessaire pour la formation de cette plaque variait pour un individu de grandeur moyenne entre 55 et 90 secondes et il était remarquable que si l'on empêchait l'animal d'achever sa tâche en touchant le pied par un objet quelconque, la plaque terminale était moins solide, moins épaisse et le fil plus mince. Plusieurs fois en touchant chez un même animal après des laps de temps déterminés d'avance j'obligeais l'animal de faire entrer son pied dans ces coquilles. Des mesures microscopiques des diamètres des fils, qu'il avait filés, me prouvèrent alors, que les carrés de ces diamètres étaient proportionnés au temps que l'animal avait employé.

J'ai observé encore souvent que le pied par un mouvement latéral élargissait la plaque ter- 
minale sans qu'il me fût possible de deviner le pourquoi de cette manoeuvre. Alors sur cette plaque étaient attachés deux fils dont le dernier pour. lequel l'animal avait employé plus de temps, était aussi le plus fort. Ordinairement quand l'animal avait terminé une plaque il retirait le pied et allait former ailleurs une autre, loin de la première et dans une autre direction. Cependant il arrivait quelquefois que le pied après avoir attaché un fil à une plaque terminale se glissait dans la même direction un peu plus loin, formant de cette manière deux fils qui étaient étendus à peu près parallèlement.

Il sera inutile d'ajouter que quand nous fîmes la même expérience avec des individus de moindres dimensions, la plaque terminale était toujours en proportion avec la grandeur de la fente transversale. Il restait à étudier par grossissement plus fort la constitution de la plaque terminale. La méthode de préparation était toute simple. Traitées pendant deux heures par l'alcool de 700, les plaques terminales avec une partie du fil furent colorées dans le picrocarminate d'ammoniaque pendant 10 heures. Alors par de l'alcool $10 \%$ l'acide picrique fut dissous et enfin j'ai durc ${ }^{\mathrm{i}}$ peu à peu par de l'alcool jusqu'a $96^{\circ}$ à $100^{\circ}$. La préparation fut monteé dans le baume de Canada après l'avoir traitée par la térébenthine. Etudiée par F et K. Zeiss. Ocul. I la plaque terminale est constituée par des milliards de petits granules, qui sont distribuées de la manière la plus irrégulière et qui sont fortement colorés. Ici ils sont très petits de sorte qu'ils ne sont à distinguer que par Zisiss. K. Imm., ailleurs ils sont plus grands et les grands serrés les uns contre les autres. Quelquefois des attroupements de granules relativement grands alternent avec des granulations très petites, de sorte qu'il paraît comme si une matière glutinante non granuleuse unissait ces attroupements. Pourtant là où je doutais qu'une matière non granuleuse les unît, il m'a été possible en employant des systèmes d'immersion, d'y distinguer une matière granuleuse très fine. Donc la plaque terminale du fil de byssus doit son origine dans son ensemble à une matière granuleuse dout les granules sont de grandeur très divergente.

Dans toutes mes préparations on voit diverguer du fil du byssus dans la plaque terminale trois ou quatre, quelquefois cinq ou six fils plus minces, comme autant de rayons. Examinés par un grossissement fort ces fils minces aboutissent aux attroupements décrits plus haut par des traînées réfringentes et granuleuses. D'où je conclus que ces fils minces qui rayonnent vers un seul point pour former un seul fil du byssus et pour la même raison ce fil de byssus lui même, sont formés par l'agglutinage de granules de diverse taille. Les granules de grande taille à leur tour sont formés par la conjonction de granules de petite taille, qui comme nous l'avons démontré dans le chapître précédent, débouchent entre les cellules épithéliales du sillon.

Il est évident que la formation $d u$ byssus d'après notre manière de voir est des plus simples. Les parois et les lamelles de la cavité byssifère sécrétant incessamment une matière byssogène, il sera formé entre les lamelles de la cavité des plaques ou des lamelles de la racine (Wurzellamellen A. MüLler). Ces lamelles dans la partie antérieure de la cavité, qui chez Mytilus est plus étroite, s'unissent et se soudent les unes aux autres et, puisque l'orifice est encore plus étroit, ces lamelles tordues et repliées de mille façons, formeront le tendon rond de RÉAumur, notre tronc de byssus. Des coupes transversales pratiquées sur le tronc de byssus de Mytilus démontrent la vérité de cette hypothése de la manière la plus péremptoire, et là où la cavité byssifère n'a pas un orifice si prononcé et où les lamelles sont placées plus irrégulièrement, comme par exemple chez Dreyssena polymorpha et Modiolaria discors on voit, dans une coupe transversale du tronc, des lamelles de matière byssogène tordues irrégulièrement qui alternent avec des lacunes. (Voyez fig. 36 et 37 ).

Puisque le sillon ventral du pied aboutit dans la cavité byssifère, et que des glandes de la même espèce sécrètent là aussi leur matière byssogène, il va sans dire, que chaque fil de byssus qui est formé dans le sillon est soudé immédiatement au tronc de byssus. L'exposé de RÉAumuR cité plus haut comme si la matière byssogène du sillon coulait dans la cavité byssifère pour former là le tronc dę byssus, est donc tout à fait incorrect. Le tronc de byssus étant comme 
fixé dans l'orifice de la cavité, il en suit que ces fils seront ordinairement fixés à la même face. antérieure du tronc. Toutefois il arrive qu'une série de fils sont attachés à un côté et une autre série au côté opposé. Un examen minutieux me montrait alors que l'animal, avant de filer la. seconde série, avait tordu le trone de byssus de $180^{\circ}$, évidemment à la suite de circonstances imprévues, p. c. séparation violente, etc.

Comme déjà Réaumur a observé, le tronc de byssus croît comme un cheveu c'est à dire: la sécrétion continuelle de matière byssogène entre les lamelles de la cavité byssifère fait allonger le tronc de byssus et le pousse constamment en dehors de l'orifice, d'où il suit que les fils de byssus les plus jeunes sont toujours soudés à la partie la plus proximale du trone de byssus, une observation qui a été faite déjà par MüLler.

- Nous avons déja discuté l'opinion de MüLler, qu’il y aurait une matière agglutinative et une matière byssogène, la première étant formée dans la cavité byssifère, l'autre dans le sillon. L'étude histologique et anatomique des organes byssogènes s'y oppose. La classification artificielle des différentes espèces de byssus du même auteur devait nécessairement aboutir à une. exposition aussi spécieuse de la manière dont est formé le byssus chez les différentes classes ${ }^{1}$ ) et en vue des résultats obtenus dans la première partie, nous croyons nous pouvoir abstenir d'une critique de ces opinions.

\section{Sur l'introduction de l'eau dans le système circulatoire des Lamellibranches.}

La question de l'introduction de l'eau dans le système circulatoire des Mollusques en génér al et des Lamellibranches en particulier a soulevé dans les derniers temps de vives et nombreuses discussions. MM. Kolluman ${ }^{2}$ ), Griesbach ${ }^{3}$ ), Carrik̀re $^{4}$ ) et Barrois ${ }^{5}$ ) ont publié d'intéressants travaux sur ce point si douteux. Si Kollman et Griesbach soutiennent la présence des pori aquiferi (Della Chiaje. Voyez pag. 22) placés ordinairement sur la carène du pied, par lesquels de l'eau ambiante est introduite dans le système circulatoire, CARrik̀re et Barrois au contraire

1) Müluler. 1. c. Erklärung der Formen der Byssus, pag. 34 .

2) J. Kollman. Der Kreislauf des Blutes bei den Lamellibranchiern, den Aplysen und den Cephalopoden. Zeitschrift f. W. Zool. XXVT. p. 87.

Le même. Die Bindesubstanz der Acephalen. Archiv f. Mikrosk. Anat. XII 1876. pag. 558:

Le même. Ueber Verbindungen zwischen Coelom und Nephridium. Festschrift. Basel 1882.

Le même. Pori aquiferi und Intercellulargänge etc. Verhandl. der Naturf. Gesellsch. in Basel. VII. 2. Heft.

3) H. Griesbacr. Ueber das Gefässsystem und die Wasseraufnahme bei den Najaden und Mytiliden. Biol. Centralblatt. II. Bd. $\mathrm{N}^{\circ}$. 10. Seite 306 .

Le même. Sous le même titre. Zeitschr. f. W. Zool. XXXVIIr. Bd.

Le même. Die Wasseraufnahme bei den Mollusken. Biol. Centralblatt. II. N. 18. pag. 573.

Le même. Die Wasseraufnahme bei den Mollusken. Zool. Anzeiger. 1883. Seite 515.

Le même. Zur Frage: Wasseraufnahme bei den Mollusken. Zool. Anz. 1884. Seite 169.

4) Justus Carrière. Vorläufige Mittheilung. Sitzung 4. Mai 1878 des Würzb. physik. med. Gesellschaft.

Le même. Ueber die Drüsen im Fusse der Lamellibr. Arb. zool. zoot. Instit. Würzb. Bd. V. p. 56.

Le même. Haben die Mollusken ein Wassergefässsystem. Biol. Centralbl. I. pag. 677.

Le même. Die Fussdrüsen der Prosobranchier und das Wassergefässystem der Lamellibranchier und der Gastropoden. Archiv f. mikrosk. Anat. XXI. pag. 387.

Le même. Das Wassergefässsyst. der Lamellibr. und Gastrop. Zool. Anz. 18\%1. No. 90.

Le même. Die Wasseraufnahme bei den Mollusken. Zool. Anz. 1883. $\mathrm{N}^{\circ} .183$.

Le même. Die embryonale Byssusdrüse von Anodonta. Zool. Anz. 1884. N .158.

5) Outre les écrits mentionnées dans la partie $\mathrm{A}$.

Barrois. Les pori aquiferi et Jes cuvertures des glandes byssogènes. Lille 1883.

Le même. Sur l'introduction de l'eau etc. Lille 1884. 
soutiennent qu'il n'y a jamais plus qu'une seule ouverture qui alors n'est autre chose que l'orifice des glandes byssogènes et ne communique dans aucun des cas observés jusqu'à présent par eux avec le système circulatoire.

Dans une notice préliminaire ${ }^{1}$ ), en publiant quelques résultats qui sont exposés dans le chapître A, je me suis déjà prononcé sur l'absence de pori aquiferi chez les Unio, les Anodonta, les Mytilus et les Arcidae. Dans une publication récente LANkester ${ }^{2}$ ) sur des séries de coupes transversales pratiquées par le pied des Anodonta et de Solen »could find no such pores as have from $\gg$ time to time been described nor any break on the epithelial clothing of the foot which could »serve as an entrance to the sub-epithelial vascular spaces«. Cet auteur se declare donc partisan de l'opinion de CARrit̀re, de Barrois et de la mienne, qui n'admettent pas une introduction de l'eau ambiante par des pores aquifères.

Je me propose de donner à présent un exposé de mes recherches sur ce point si disputé.

GrIesbach dans son mémoire intitulé: »Über das Gefässsystem und die Wasseraufnahme bei den Najaden und Mytiliden« nous donne un exposé détaillé de quelle manière il a trouvé trois pori aquiferi sur la carêne du pied de Unio et de Anodonta, tandis que KollumanN ${ }^{3}$ ) n'en a vu qu'un seul, comme tous les auteurs avant lui, excepté von BAER ${ }^{4}$ ) qui en a vu premièrement. un seul, quelque temps après trois, puis neuf à dix qui cependant sont considéré par Griesbach comme des déchirures. Pour découvrir les pores Griesbach a suivi la méthode suivante. Rapidement il prit hors de l'eau un des animaux dont le pied était grandement allongé hors de ses coquilles et pressant les coquilles, il empêchait de cette façon l'animal de retirer le pied. Qu'est ce qui arriva alors? $\gg$ Es traten an den verschiedensten Stellen (nous cursivons) die 》bekannten Wasserstrahlen auf. Drei Strahlen zeichnen sich durch ihre Kürze von den anderen 》uus. Sie treten, wemn überhaupt unveränderlich einer auf der Spitze des Fusses und zwei dicht »neben einander ungefähr auf der Mitte der Fussschneide auf. Selten sieht man alle drei Strahlen »uggleich, meistens erscheint nur einer und zwar der, welcher aus der dritten Oeffnung von vorn 》aus gerechnet hervortritt, oder diese und die erste der drei Oeffnungen werfen zugleich Wasser »aus. Manchmal sieht man aus der Fussschneide gar keinen Wasserstrahl austreten, wenn man $\gg$ dann den Fuss sanft an der Stelle, wo die mittleren Oeffnungen liegen, zwischen zwei Finger 》drückt, so seht man oft (nous cursivons) wie Wasser aus der mittleren grössten Oeffnung nicht. »etwa strahlenartig entleert wird, sondern kaum sich über die Ränder erhebend nur überfluthet«.

Nous avons répété les mêmes expériences avec des animaux vivants. Pressant le pied allongé entre les deux coquilles nous avons vu les mêmes jets d'eau que GRIEsBaCH a observés et cela sur plusieurs endroits de la carêne du pied, mais en pressant le liquide, qui se trouvait dans les grandes lacunes $\mathrm{du}$ pied, nous avons forcé la couche épithéliale et le liquide débouchait par plusieurs jets. Aussi d'après notre opinion Griesbach a forcé également la couche épithéliale. Si la déchirure était très petite le jet d'eau était relativement bien fort, mais à mesure que deux ou trois de ces petites déchirures avoisinantes s'unissaient dans un seul trou béant le jet d'eau perdait en hauteur ce qu'il gagnait en largeur.

Cette expérience est notamment en parfaite concordance avec celle de Griesbach lui-même, que les jets d'eau étaient visibles »an der verschiedensten Stellen« et que trois d'entre eux se distinguaient 》durch ihre grössere Dicke und ihre geringere Kraft und in Folge dessen durch ihre Kurze«. Cependant chez moi ces trois jets d'eau n'étaient pas toujours au même temps visible. Et encore cette expérience est la même que celle de Griessach, comme est demontré par ses propres paroles citées en haut »Selten etc.« Quelquefois, poursuit l'auteur, il ne voyait pas un seul jet d'eau

i) Catrie. 1. e. Zool. Anz. 4883. pag. 560.

2) Lankester. Zool. Anz. 1884. No. 170. pag. 343.

3) Kollmann. Pori aquiferi etc. pag. 8.

4) Von BAer. Bemerkungen etc. Frorieps Notizen. XII. N. 1. Seite 6. 
mais s'il pressait alors l'endroit où devait être sitné le second et le troisième orifice présumé, il observait qu'un liquide, qu'il dit être de l'eau, »s'écoulait lentement« de cet endroit. Alors pas de jet d'eau, mais »l'eau s'écoulait lentement«. J'ai observé encore plusieurs autres choses non moins remarquables.

En pressant très doucement, ordinairement je ne voyais absolument pas un simple jet d'eau. Si l'animal me forçait, en tâchant de retirer le pied, de presser plus fortement, álors les jets d'eau commençaient à apparaître, et si je pressais par rhytmes, les jets d'eau suivaient les mouvements rhytmiques. Quand, en pressant doucement les coquilles, je ne voyais pas de jets d'eau et que je pressais alors quelque endroit entre les doigts, je voyais s'écouler à chacun de ces endroits lentement une quantité de liquide: c'est à dire en pressant entre les deux doigts, je forçais en ces lieux tout simplement la couche épithéliale.

Est-il permis de conclure de ces expériences que le long de la carêne du pied il y ait plusieurs orifices? Je crois que mes expériences me donnent le même droit pour cette conclusion que Griesbach a pour ses deux pores aquifères sur la seconde moitié de la carêne du pied.

Mais quel naturaliste oserait conclure de ces expériences grossières qu'il existe des pores par lesquels l'eau ambiante est en communication avec le système circulatoire?

Aussi Griesbach a fait usage de la méthode »der Selbstinjection«. Des Anodonta furent placés dans de l'eau qui était colorée par le vert de Jode. Après la mort de l'animal le pied était toujours coloré vert extérieurement et intérieurement, dit l'auteur distingué, ce qui concorde avec les résultats de mes expériences que j’ai répétées plusieurs fois. 》Aus diesem Versuchen geht $\gg$ deutlich vor, das Wasser in dem Organismus aufgenommen wird« continue $\mathrm{Mr}$ le Dr. GrIEsBACH 1) 》und zwar allem Anscheine nach durch den Fuss.« Je puis admettre ces deux sentences, sous une restriction que je formulerai plus tard, seulement, que cette introduction ait eu lieu par des pores aquifères, voilà ce qui est encore très-douteux.

Pour observer l'introduction directe de l'eau dans le pied par des orifices, Griesbach distribuait »Magnesia usta« dans l'eau et il observait que les cils vibratiles de la couche épithéliale des orifices présumés faisaient mouvoir la poudre.... \und Spuren desselben drangen mit den »Wasser ein«. Donc ce n'étaient que de très faibles quantités »Spuren« qui s'introduisaient dans ces orifices. 》Ebenso bei anderen Versuchen Jodgrün und Karminpulver«. Cependant Griesbach n’a jamais pu observer dans ces expériences qui furent faites sur des An odonta jeunes (de 2,3-3,5 centimètres) 》ein Herausschnellen der Farbstoffpartikelchen oder einen Wasserauswurf aus den $\gg$ Pori aquiferi«. Néanmoins ces expériences sont d'après son opinion telles »dass die Frage nach $\gg$ den Wasseraufnahme positiv beantwortet wird«.

Quant à moi, je n'ai jamais réussi à observer une introduction directe soit de poudre de earmin, soit de Magnesia usta quelque envie que j'eusse eue à la voir. Alors, après mille tentatives vaines, j'ai suivi un autre chemin qui, d'après mon opinion, devrait donner les résultats les plus positifs. J'ai suivi la méthode des coupes transversales à l'aide du microtome. Des individus de Unio et de Anodonta d'une grandeur plus que normale (Unio batavus et Anodonta ventricosa) moururent, lentement asphyxiés dans un bassin dont l'eau ne fut pas renouvelée. Chez la plupart, le pied débordait alors les coquilles. Puis le pied fut coupé et mis durant cinq heures dans l'acide picrique de Kleynenberg. Renouvelant constamment l'alcool de $70^{\circ}$ dans lequel ils furent alors conservés, jusqu'à ce qu'il n'était plus coloré jaune, je fis usage du picrocarminate d'ammoniaque pour colorer pendant 10 à 14 jours. Dissolvant plus tard l'acide picrique par l'alcool de $40^{\circ}$, je durcissais successivement par l'alcool de $70^{\circ}$ et l'alcool absolu. L'objet restait alors dans l'alcool absolu en le renouvelant quelquefois jusqu'à que le durcissement était assez complet. Après l'avoir traité par le chloroforme (Méthode de Gresbrecht) j'employais un mélange de paraffine et de vaseline comme masse encastrante. De

1) Griesbach. Z. f. w. Z. XXXVIII. 1. e. pag. 30. Separat-Abdruck. 
cette manière j'ai pratiqué des séries de coupes transversales sur 3 et 4 exemplaires de A nodonta ventricosa et Unio batavus.

Dans aucun endroit des centaines de coupes transversales ${ }^{1}$ ) l'épithélium de la surface du pied n'offre la moindre solution de continuité. Donc chez les espèces sus-nommées il n'existe pas de pori aquiferi.

Griesbach dans une publication récente ${ }^{2}$ ) a émis des doutes sur la valeur de la méthode des coupes transversales employée par CARRIÈre, TH. Barrors et moi et croit en particulier devoir attribuer la cause que je n'ai pas pu voir le petit sac décrit par Carrière comme étant une cavité byssifère dégradée dans le pied des Anodonta, à ma méthode de préparation. CARrì̀re. sur trois à quatre exemplaires qu'il a examinées n'a observé ce sac qu'une seule fois. S'en suitil qu'un autre naturaliste sur deux fois trois exemplaires aura aussi deux fois cette bonne fortune? N'est-il pas possible qu'il ne l'a pas une seule fois? 'J'ai exposé en haut ma méthode de préparation. Si vraiment les pori aquiferi existent serait-il alors possible que des ouvertures de 1-3 millimètres de longueur (d'après les mensurations de Griesbach lui même) puissent échapper six à sept fois de suite dans des séries de coupes dont les plus épaisses mesurent à peine $\frac{1}{80}$ d'un millimètre, mais dont la plupart sont plus minces ( $\frac{x}{6}$ d'un millimètre). Serait-il possible que: la méthode de préparation décrite plus haut eût eu une telle influence, eût contracté tellement. les lèvres de l'orifice présumé que la couche épithéliale paraît être continue au lieu d'avoir. un manque de continuité.

Pour faire une contre-expérience j'ai coupé le pied linguiforme de la moule commune aussi près de la bosse de Polichinelle que possible, je l'ai jeté immédiatement dans l'alcool absolu et: je l'ai conservé ainsi pendant huit jours, renouvelant chaque jour l'alcool. Puis traité par le chloroforme et encastré dans un même mélange de paraffine et de vaseline, toujours de la manière: la plus péremptoire on pouvait voir distinctement dans des coupes transversales le sillon ventral. avec son canal semi-lunaire.

Griesbach, désapprouvant la méthode des coupes est au contraire plein d'éloges pour sa propre méthode de la »Selbstinjection« et me donne l'avis de faire geler les objets et d'employer. l'acide osmique. Quant à la méthode de la »Selbstinjection« il est utile de faire remarquer que: Flemming ${ }^{3}$ ) et Nalepa ${ }^{4}$ ) ont désapprouvé vivement l'emploi des couleurs à aniline, puisque ces: couleurs par l'osmose entrent dans les tissus sans prouver cependant qu'il y a introduction par des orifices présumés.

Comme je l'ai déjà prononcé en haut j'ai vu que de l'eau colorée par le vert. de Jode pénétrait dans les tissus du pied des A nodonta et Unio, mais je faisais alors une: restriction. C'est ici l'endroit convenable de donner comme mon opinion que cette eau colorée: y pénetrait tout simplement. par osmose et non à cause d'ouvertures qui se trouveraient sur la. carène du pied.

Quant à la méthode de gélation qui est recommandée si hautement par Griesbach je me borne à citer deux auteurs, $K_{\mathbf{E Y}}$ et RETZIus ${ }^{5}$ ) qui, se basant sur leurs expériences, nous démontrent que les tissus subissent quelquefois de telles déformations qu'on ne peut en aucune façon se fier aux résultats de diverses études histologiques et microscopiques. Je crois au contraire que quand il s'agit de prouver une discontinuité de la couche épithéliale chez les Mollusques c'est

1) Catrue. Vorläufige Mittheilung. Zool. Anz. 1883. pag. 561.

2) Griesbach. Zool. Anz. 1884. Seite 169.

3) Flemming. Bemerkungen hinsichtlich der Blutbahnen etc. Zeitschrift f. w. Zoöl. XXXIX, pag. 143.

4) Nalepa. Die Intercellulairräume des Epithels etc. LXXVII. Bd. der Sitzber. der K. Akad: der Wiss. I. Abth. Nov. Heft 1883. Seite 1184.

5) KEX et Retzlus. Om Frysnigsmetodens an van dande vid histologisk teknik. Nordisk medicinsk Arkiv. Bd. VI. 1874 
justement la méthode de gélation qu'il ne faudra jamais employer à cause des déchirures qui seront toujours occasionnées par la crystallisation de l'eau, dont sont imprégnés tous les tissus des ces animaux.

Je crois volontiers que le traitement par de l'acide osmique a ses avantages, mais en vérité est-il vraisemblable qu'un de ces avantages serait de démontrer l'existence de pores aquifères longues de 1 à 3 millimètres, alors que la méthode de préparation que j'ai employée n'a jamais offert la moindre solution de continuité de la couche épithéliale du pied? Griesbach après avoir constaté (?). l'introduction de l'eau chez les Najades a ensuite étudié chez Mytilus edulis et Dreyssena polymorpha la même question et il là étudiée par... des sondes ${ }^{1}$ ), c'est à dire il a introduit par la fente transverse, le commencement du sillon, une sonde de caoutchouc de l'épaisseur d'une aiguille à tricoter. Et puisque cette sonde entrait dans les vaisseau sanguin (Voyez fig. 11) (évidemment en perforant la couche épithéliale du sillon semi-lunaire), la fente transverse est décrétée un 》porus aquiferus et les recherches de KolLmans ${ }^{2}$ ) sur les $\gg$ Wasserröhren« de Pecten, Spondylus et Mytilus et. de Sabatier ${ }^{3}$ ) sont citées comme autant de preuves pour la justesse de ses observations et conclusions.

Il sera bien inutile après les recherches minutieuses de Tullberg et de Carrière, pour ne pas parler des miennes, de faire la critique du procédé employé par Grıesiach. Si l'auteur, au lieu de faire usage d'une sonde, avait commencé par pratiquer des coupes transversales aussi minces que possible, par la partie antérieure du pied de Mytilus il aurait pu mesurer que la distance entre la face supérieure de la couche épithéliale et le bord supérieur du vaisseau sanguin médian (Voyez fig. 11) n'est que de 0,12 millimètre et que l'introduction d'une sonde ordinairement est plus que suffisante pour faire un trou béant dans la couche épithéliale.

En effet chez toutes les espèces étudiées sur les organes byssogènes et nommées dans la partie A de ce mémoire, nous n'avons trouvé en aucun endroit une solution de continuité de la couche épithéliale qui revêt le sillon qui mène à la cavité byssifère et cette cavité elle-même.

Tous les orifices et toutes les ouvertures dont nous avons constaté la présence étaient les conduits de glandes byssogènes plus ou moins dégradées. Et chez les espèces où il n'y avait pas la moindre trace de glandes byssogènes, les Yoldia, Nucula, Leda, Mya, Anodonta et Unio, il n'y avait pas non plus les moindres traces d'orifices, qui fussent en communication avec le système circulatoire.

Si nous résumons enfin les résultats obtenus par Carrière, Barrois et les miens sur l'introduction. de l'eau dans le système circulatoire, il est évident, d'après notre opinion, que s'il y a introduction de l'eau ambiante, cette introduction n'a pas lieu par des pori aquiferi, les orifices constatés ainsi nommés étant les ouvertures de glandes byssogènes plus ou moins dégradées.

Il nous reste en dernier lieu à discuter de quelle manière les pieds des Mollusques en général et des Lamellibranches en particulier peuvent se gonfler si énormément qu'ils débordent par deux ou trois fois leur longueur habituelle hors des coquilles.

CARRIÈre ${ }^{4}$ ) d'une manière concise et précise a déjà éclairci la question. Avec cet auteur je suis d'opinion que la sang contenu dans la système vasculaire et lacunaire de ces animaux suffit pour faire gonfler le pied. Cette opinion a trouvé un défenseur énergique en Lankester ") qui

1) Griesbach. Ueber das Gefässsystem etc. pag. 33.

2) Kollmann. Z. f. W. Zool. XXVI. pag. 97.

3) Sabatien 1..c. pag. 458-463. (Voyez aussi ma notice préliminaire Zoöl. Anz. 1883. l. c.

4) Carrière. Die Fussdrüsen der Prosobranchier etc. pag. 427 sqq.

5) Lankester. 1. e. Zool. Anz. 1884. p. 344 
nous donne les résultats d'expériences et d'observations faites il y a une douzaine d'années. Cet' auteur nous les décrit comme suit: $\gg$ An examination of the living specimens of Solen legumen »made by me at Naples in 1872 was absolutely convincing as to the fact that on that Lamelli》branch the blood-fluid is under no circumstances shed from any pores or apertures in the 》animal's body, so long as the surface is uninjured.

$\gg$ It was equally conclusive as to the fact that complete destension of the foot is produced in $\gg$ that animal without any admission of water in the vascular system, by the »simple mechanisme of a rapid flow of the deep-red-coloured blood from the mantle and body $\gg$ of the Lamellibranch into its foot.

$\gg$ It was easy to observe the to-and-fro movement of the blood when a specimen of Solen 》legumen was taken out of the water and held in the hand, owing by the deep red colour $\gg$ of the blood caused by its corpuscles. The whole vascular system can be readly traced in this 》Lamellibranch owing to the transparency of the tissues and the red colour of the blood.

$\gg$ With regard to the Gastropoda the same kind of evidence is afforded by Planorbis $\gg$ corneus. The red-colored haemoglobinous fluid is under no circumstances shed from the $\gg$ body of that animal unless its surface is wounded. Similarly there is no evidence of a dilution $\gg$ of the red-coloured fluid (such as would be afforded by its requiring a paler tint) when the $\gg \mathrm{Planorbis}$ is made to expand and retract its foot repeatedly. Were water admitted to the $\gg$ blood in the act of expansion of the foot, the repetition of this act would necessarily lead to »a dilution of the haemoglobin and a diminution of the red colour of the blood-fluid «.

Certainement les faits observés sont frappants et les raisonnements concluants. Cependant il est bon de remarquer qu'ils heurtent de front les recherches de Arrred NALEPA ${ }^{1}$ ) sur la structure de l'épithélium des Gastropodes pulmonés. Ce naturaliste outre qu'il a vu entre les cellules épithéliales les orifices de glandes, qui sécrètent une matière muqueuse et calcaire, a observé de petits pores qu'il croit être les orifices d'espaces intercellulaires.

Afin de démontrer leur continuité avec le système sanguin, l'auteur a employé la méthode du Dr. Altman: imprégnation par une matière grasse et traitement par l'acide osmique. De cette manière il a rempli les espaces intercellulaires et les vaisseaux sanguins avec lesquels ceux-ci étaient en continuité, avec une matière grasse noirâtre.

Pour affaiblir l'objection que ces pores intercellulaires peuvent être les conduits de glandes muqueuses (et grasseuses?), l'auteur distingué a fait des injections avec une masse composée de glycérine, de gélatine (colle) et de carmin, dissous dans l'ammoniaque. Les résultats de ces injections étaient que rarement la masse injectée pénétrait plus loin dans le pore intercellulaire que de deux tiers de la hauteur. »Selten dringt die Injectionsmasse über das obere Drittel der Zellhöhe.« L'auteur devait examiner plusieurs coupes avant de rencontrer une seule dans laquelle la masse injectée avait rempli toute la longueur du pore et sortait à l'extérieur. Mais il a rencontré plusieurs fois que la masse injectée s'était intercalée comme un coin entre deux ou plusieurs cellules et les avait soulevées, de sorte qu'il y avait un manque de continuité à cet endroit. »Aus dem Gesagte geht wohl deutlich hervor, dass die zwischen den Epithelzellen ge》legene Räume thatsächlich Intercellularräume sind die einerseits mit Bluträume anderseits mit $\gg$ dem umgebenden Medium in Verbindung stehen. Die Ergebnisse der hier in Anwendung ge»brachten Untersuchungsmethoden schliessen jede andere Deutung aus.« Ainsi continue l'auteur distingué.

Vraiment serait-il indubitable que le procedé d'imprégnation suivi et contrôlé par des injections d'une masse colorée a donné des résultats qui du moins chez les Gastropodes pulmonés démontrent d'une façon péremptoire que les canaux intercellulaires signalés depuis longtemps par

1) NALEPa. 1. c 
Leydig chez le Cyclas font communiquer l'eau avec le système lacunaire sanguin? Par exemple n'est-il-pas possible que les pores intercellulaires soient des conduits de glandes adipeuses ou grasseuses qui par l'emploi de l'acide osmique sont colorés d'un ton noirâtre? Il y a des naturalistes qui - et certainement non sans raisons valables - disent des injections. 》Timeo injectiones et nova ferentes.« En effet, je crois aussi qu'on doit accepter les résultats obtenus par la méthode des injections sous mainte restriction. L'auteur lui-même dit que rarement la matière injectée a rempli entièrement tout le canal et que plusieurs fois quelques cellules étaient soulevées et qu'il y avait alors discontinuité de la couche épithéliale. Si nous y ajoutons que l'introduction de l'eau ambiante dans le système circulatoire, d'après les paroles de LANKESTER est 》a somewhat startling physiological process, « qui est tout à fait contraire à l'économie de la nature, il sera évident qu'il nous faudra des observations plus satisfaisantes et plus frappantes avant que l'introduction de l'eau dans le système circulatoire soit un fait établi.

Arnhem, 1 Oct. '84. 


\section{Explication des Planches.}

Dans toutes les figures la couleur bleue désigne la couche épithéliale et les couleurs verte et rouge des glandes byssogènes.

m. b. muscles byssales.

$m$. muscles longitudinales et transverses.

m. by. matière byssale.

ov. ovaire.

c. s. cellules spermatozoides.

$n$. nerfs.

v. s. $m$. vaisseau sanguin médian.

v. s. $l$. vaisseau sanguin latéral.

t. c. tubes cilindriques qui communiquent avec le sillon.

p. $l$. pied linguiforme (Spinnfinger).

b. d. $P$. bosse de Polichinelle.

d. partie du canal alimentaire.

Fig. 1. Coupe transversale du pied linguiforme de Pecten islandicus. No. 485; gros= sissement $\frac{40}{1}$.

- 2. Coupe transversale de la même species. $N^{\circ} .518 ; \frac{40}{1}$.

- 3. Idem. $\mathrm{N}^{\circ} .565 ; \frac{40}{1}$.

- 4. Idem. $\mathrm{N}^{\circ} .763 ; \frac{40}{1}$.

- 5. Coupe transversale du pied linguiforme de Pecten groenlandicus. No. 212; Ire série; $\frac{40}{1}$.

- 6. Idem. $\mathrm{N}^{\circ} .200 ; \mathrm{II}^{\mathrm{e}}$ série; $\frac{40}{1}$.

- 7. Une partie de la coupe transversale. $\mathrm{N}^{\circ} .67$; grossie 410 diamètres pour démontrer la présence de trainées réfringentes qui constituent un fil du byssus (m. by.) qui se trouve dans le sillon (Modiolaria discors).

- 8. Coupe transversale du pied de Modiolaria discors. No. 343; $\frac{40}{1}$.

- 9. Une partie de la lamelle médiane $\alpha$ de la figure précédente. $\frac{410}{1}$.

- 10. Coupe transversale du pied de Modiolaria discors. $N^{\circ}$. 384; $\frac{40}{1}$.

- 11. Coupe transversale du pied de Mytilus edulis (I. B. Rang. 4. No. 10); $\frac{40}{1}$.

- 12. Des cellules muqueuses de Mytilus edulis (C. Rang. 2. No. 10); $\frac{690}{1}$.

- 13. Une partie du sillon avec les tubes cilindriques. Coupe transversale de Mytilus pellucidus (Série A. No. 102); $\frac{40}{1}$. 
Fig. 14. Coupe transversale du pied linguiforme de Mytilus pellucidus ( $B_{2}:$ d. 2; rang. $2 ; \mathrm{N}^{\circ} .2$ ).

- 15. Coupe transversale du pied linguiforme de Saxicava rugosa $\left(\mathrm{N}^{0} .364\right) ;{ }_{1}^{40}$.

- 16. Idem (A. No. 394); $\frac{40}{10}$.

- 17. Idem (A. No. 409); $\frac{40}{1}$.

- 18. Idem (No. 474); ${ }^{40}$.

- 19. Idem $\left(\mathrm{N}^{0} .554\right) ; \frac{40}{1}$.

- 20. Cellules glandulaires en divers phases de transformation; de Saxicava rugosa. $\frac{550}{1}$.

- 21. Vue du pied linguiforme de Arca pectunculoïdes, avec le fil du byssus.

- 22. Vue du pied linguiforme de Arca pectunculoïdes, la face droite étant découpé.

- 23. Coupe transversale de Arca pectunculoïdes (Série A. No. 173); $\frac{40}{1}$.

- 24. Idem (Série A. No. 181); $\frac{40}{i}$.

- 25. Idem (Série A. No. 204); $\frac{40}{1}$.

- 26. La partie $\alpha-\beta$ de la coupe fig. 24 par grossissement plus forte.

- 27. Vue du pied linguiforme de Dreys sen a polymorpha $\left(a b=N^{0} .391 ;\right.$ cd $=N^{0} .615$; ef $=\mathrm{N}^{0}$. 734).

- 28. Coupe transversale menée par la ligne $a^{3}$ de la fig. 27 ( $\left.\mathrm{N}^{\circ} .391\right) ; \frac{20}{1}$.

- 29. Coupe transversale menée par la ligne $c d$ de la fig. 27 ( $\left.\mathrm{N}^{\circ} .615\right) \frac{20}{1}$.

- 30. Coupe transversale menée par la ligne ef de la fig. 27 (N". 734) $\frac{20}{1}$.

- 31. Quelque cellules glandulaires pris d'une coupe transversale de Dreyssena polymorpha $\left(\mathrm{N}^{0} .248\right) \frac{410}{1}$.

- 32. Coupe transversale de la partie la plus antérieure du pied de Cardium edule (No.6. Série $\left.B_{1}\right) \cdot \frac{5 \overline{5} 0}{1}$.

- 39. Coupe transversale du pied de Cardium edule ( $N^{\circ}$. 956: Série B. ${ }^{\frac{40}{1}}$.

- 34. Idem $\left(\mathrm{N}^{\circ} .1063\right.$. B. $) \frac{40}{1}$.

- 35. Coupe transversale du pied de $\mathrm{Nucula}$ tenuis (II. rang. 3. $\mathrm{N}^{0} .7$ ) $\frac{40}{2}$.

- 36. Coupe transversale du tronc de byssus de Modiolaria discors. $\frac{100}{1}$.

- 37. Idem de Dreyssena polymorpha. $\frac{100}{1}$. 


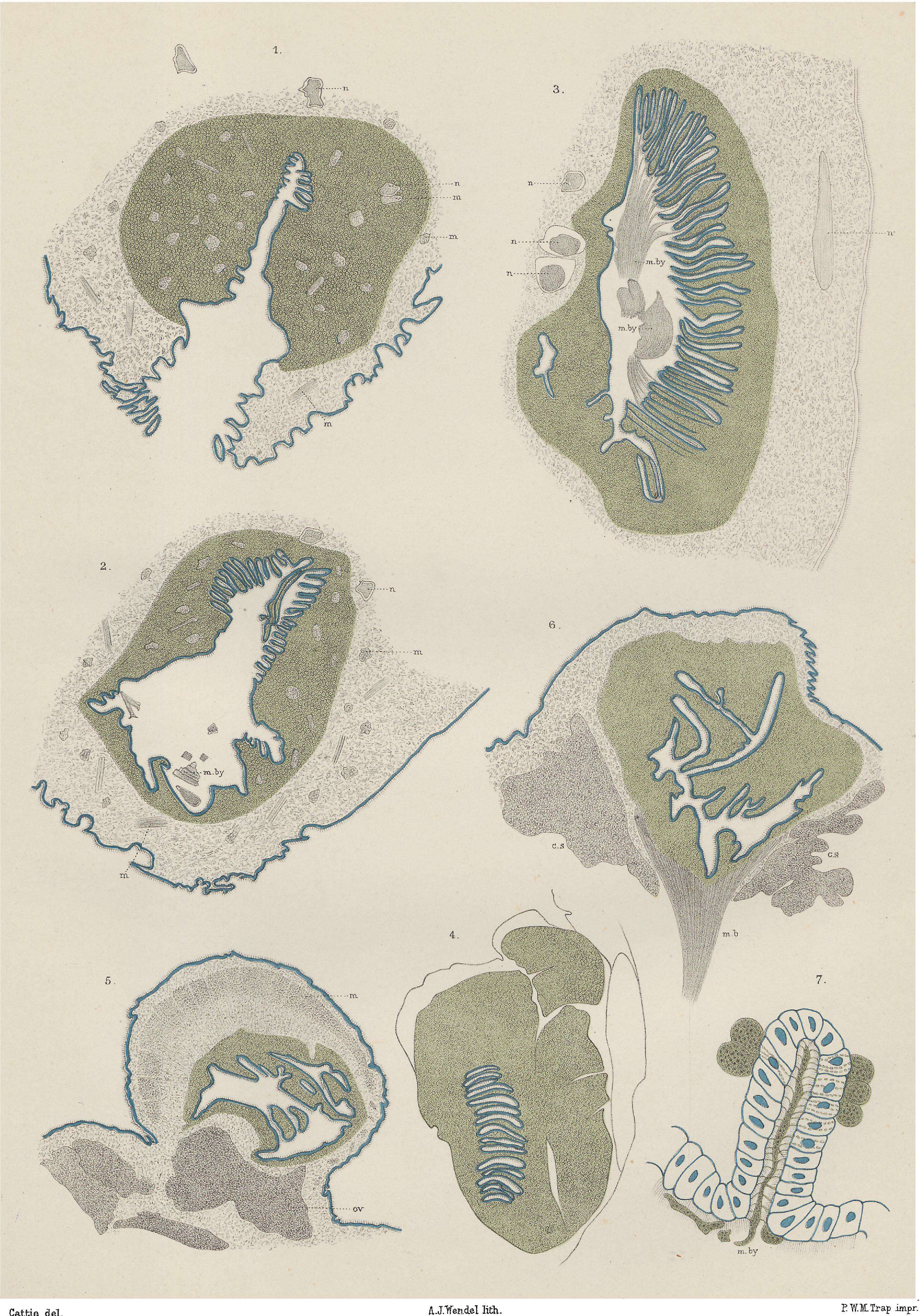




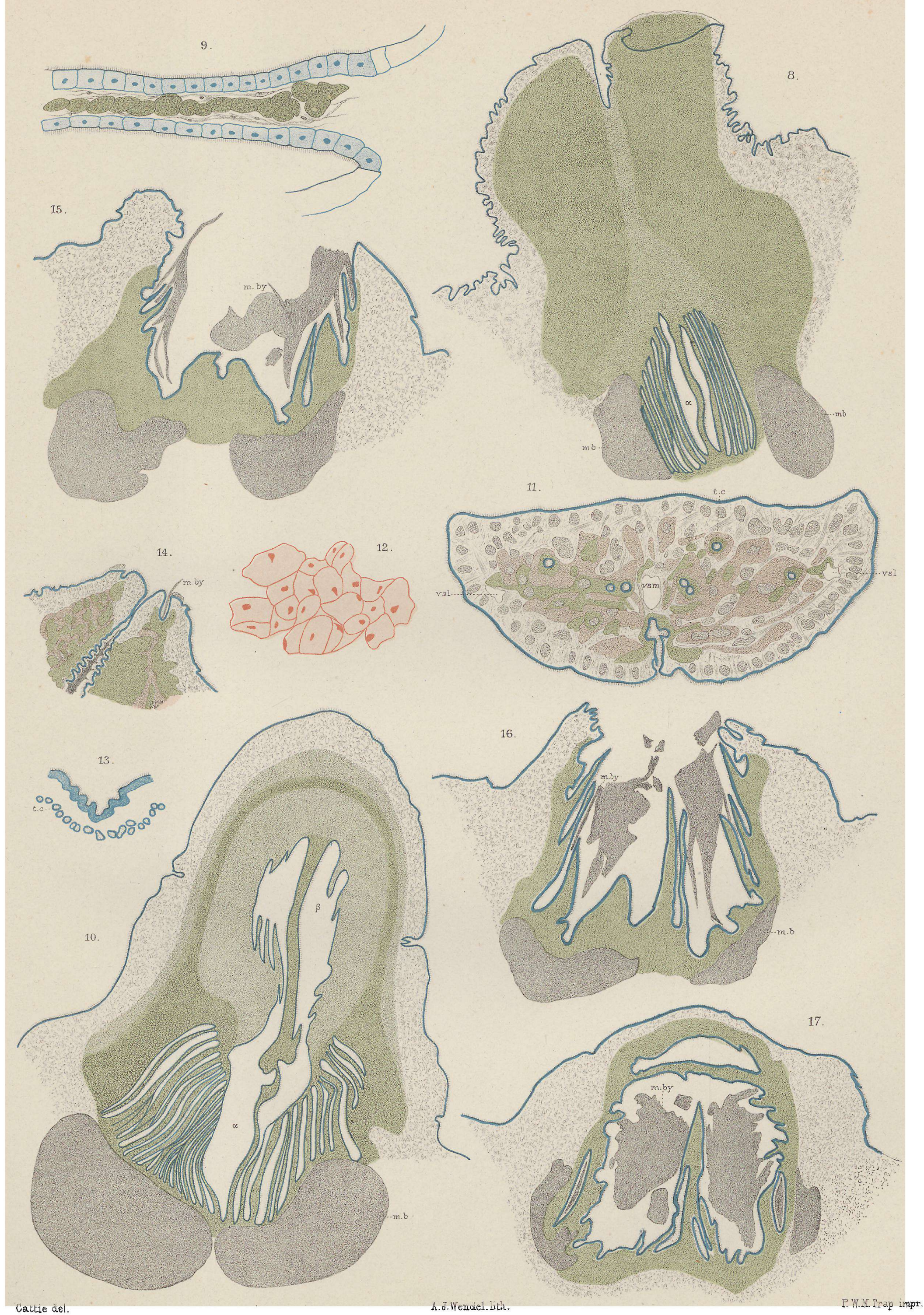




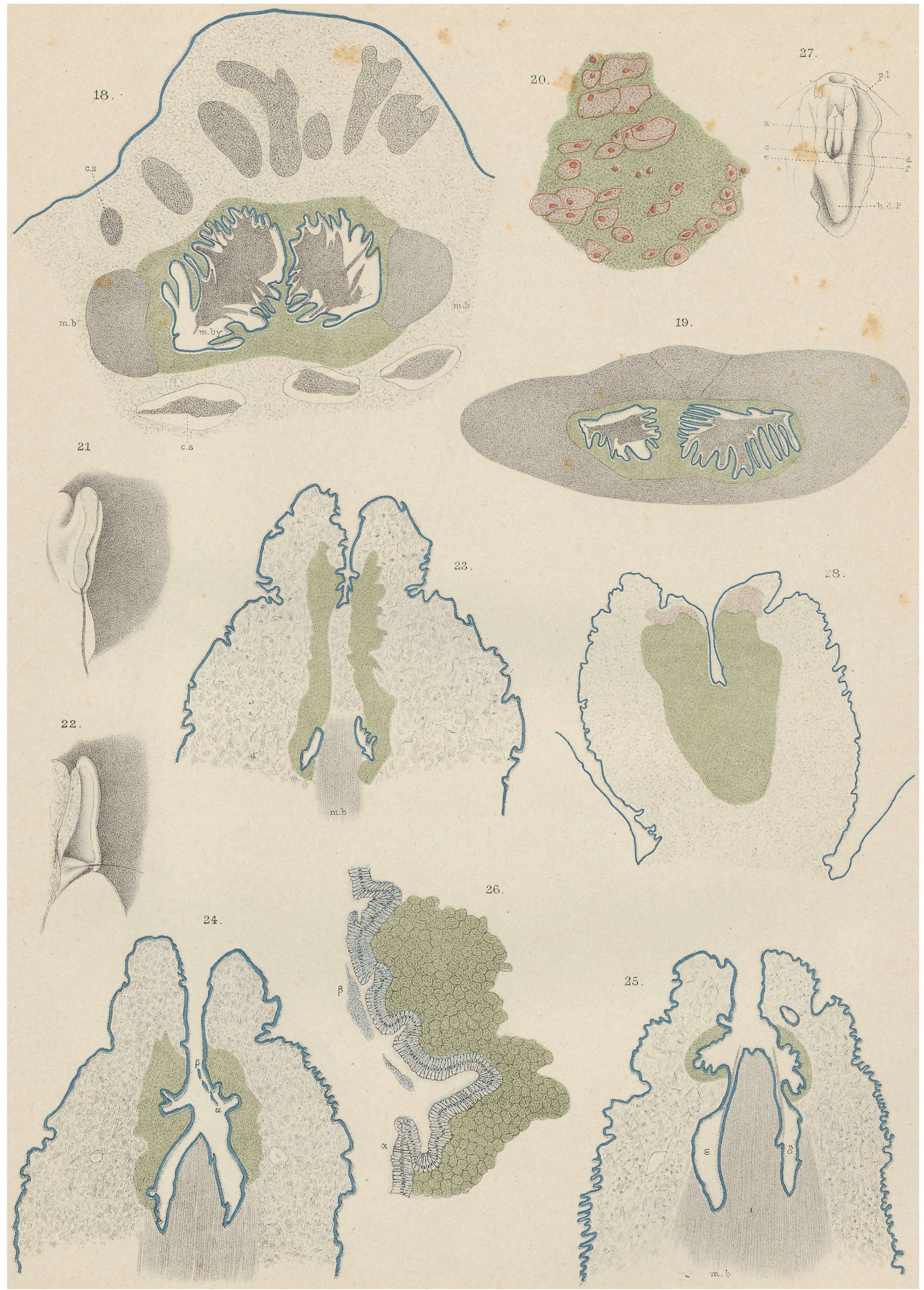




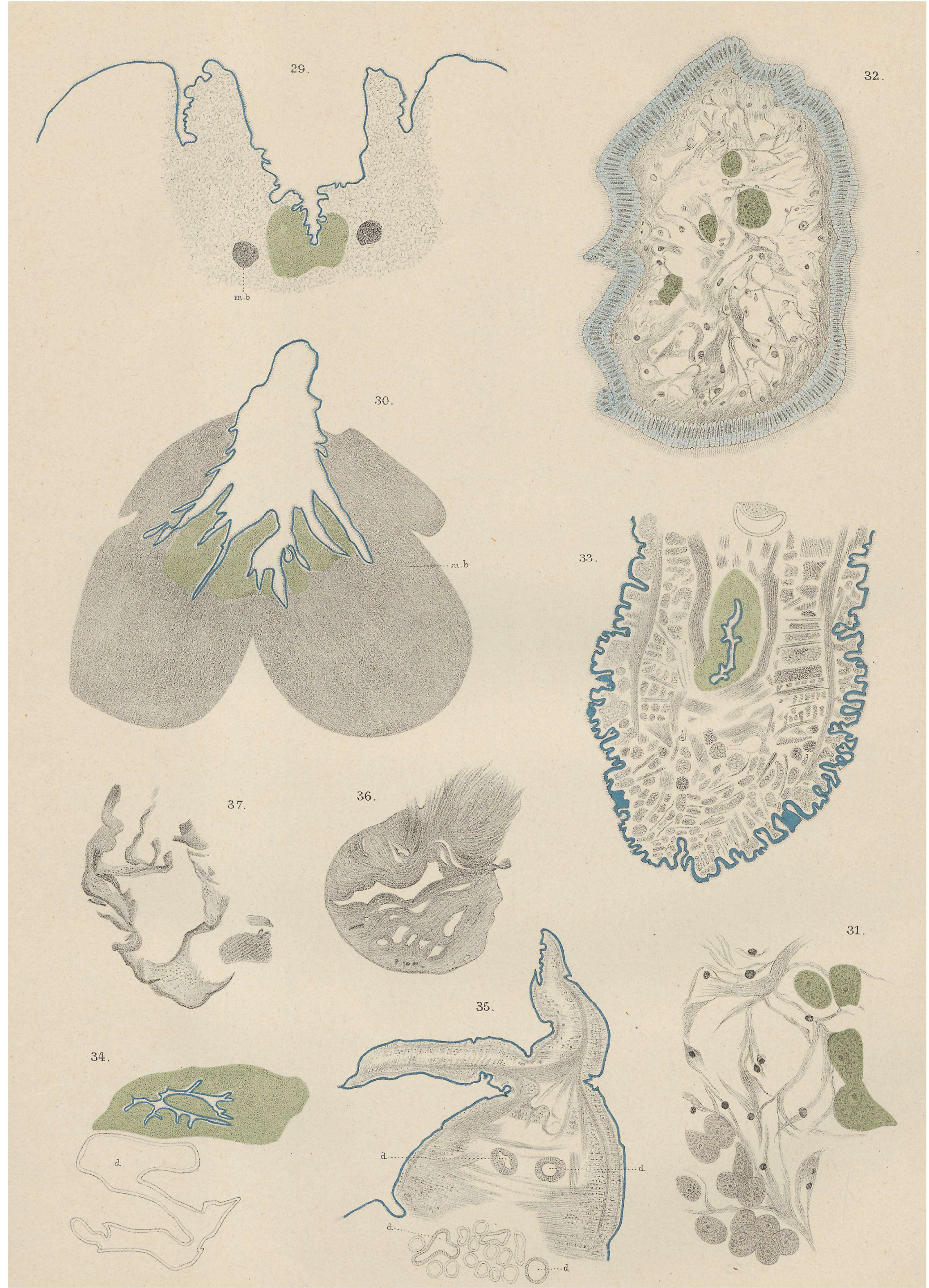

